\title{
UNIVERSITY OF HAWAIILIERARY \\ PREDICTION OF PERMEATE FLUX DECLINE IN CROSSFLOW MEMBRANE FILTRATION OF COLLOIDAL SUSPENSION: A RADIAL BASIS FUNCTION NEURAL NETWORK APPROACH
}

\begin{abstract}
A THESIS SUBMITTED TO THE GRADUATE DIVISION OF THE UNIVERSITY OF HAWAI'I IN PARTIAL FULFILLMENT OF THE REQUIREMENTS FOR THE DEGREE OF
\end{abstract}

MASTER OF SCIENCE

IN

CIVIL ENGINEERING

AUGUST 2005

BY

Huaiqun Chen

Thesis Committee:

Albert S. Kim, Chairperson

Chittaranjan Ray

Michelle H. Teng 


\section{ACKNOWLEDGEMENTS}

I would like to express my gratitude to my supervisor, Dr. Albert S. Kim, whose expertise, understanding, and patience added considerably to my graduate experience. I appreciate his vast knowledge and skill in many areas, and his assistance in writing reports (i.e. our papers and this thesis). I would like to thank the other members of my committee, Dr. Chittaranjan Ray and Dr. Michelle H. Teng, for their precious comments and valuable suggestions. Discussions with them during my thesis research was very helpful.

A very special thanks goes out to Dr. Goloka Behari Sahoo, who led me to the world of Artificial Neural Networks. He kindly provided me with direction, technical support and all his related research materials. I must also acknowledge Dr. Jim Chen for taking time out from his busy schedule to serve as my external reader and for proofreading my thesis.

I would also like to thank my family for the support they provided me throughout my entire life and in particular, I must acknowledge my husband and best friend, Qinghua Ding, without whose love and encouragement I would not have finished this thesis. 


\begin{abstract}
The capability of a Radial Basis Function Neural Network (RBFNN) to predict long-term permeate flux decline in crossflow membrane filtration was investigated. Operating conditions such as transmembrane pressure and filtration time along with feed water parameters of particle radius, solution $\mathrm{pH}$, and ionic strength were used as inputs to predict the permeate flux. Simulation results indicated that one single RBFNN accurately predicted the permeate flux decline under various experimental conditions of colloidal membrane filtrations and produced better predictability compared to those of the multiple regression method and regular multi-layer feed-forward Back Propagation Neural Network (BPNN), due to the more sophisticated training system of RBFNN. Further development of the artificial neural network approach to membrane filtration will enable the design of full or large scale processes with lab or pilot scale experiments.
\end{abstract}




\section{TABLE OF CONTENTS}

ACKNOWLEDGEMENTS .........................................................................................

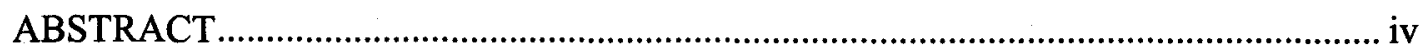

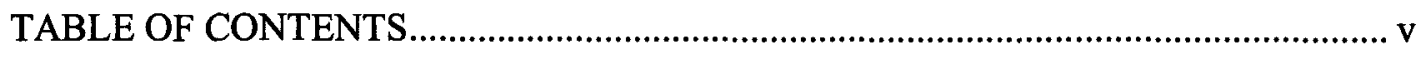

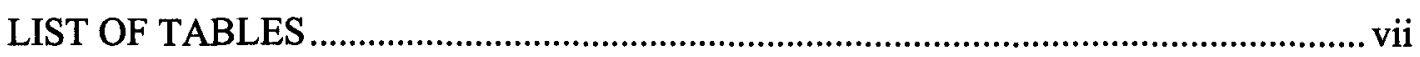

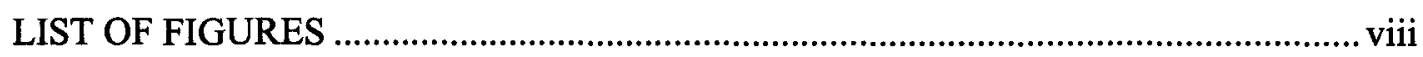

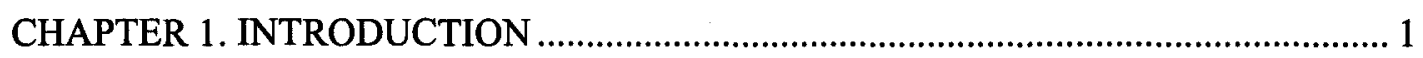

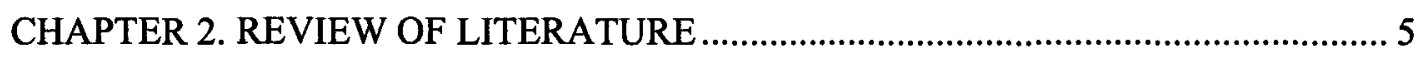

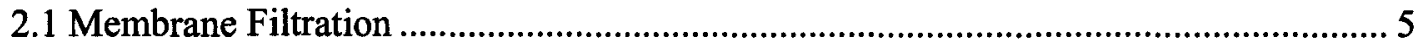

2.1.1 Membrane Types By Pore Size _.................................................................... 5

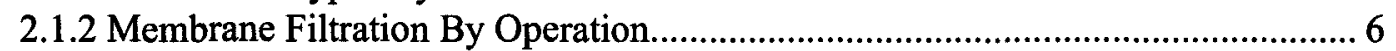

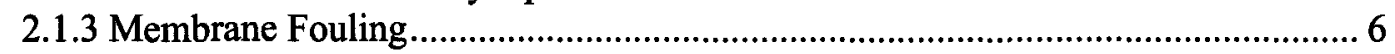

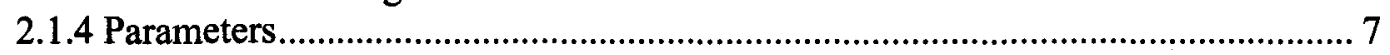

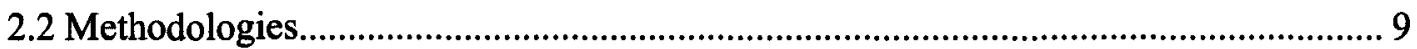

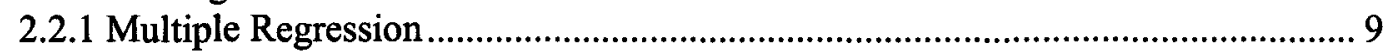

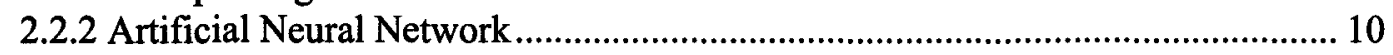

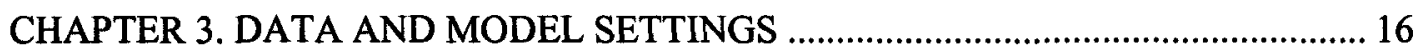

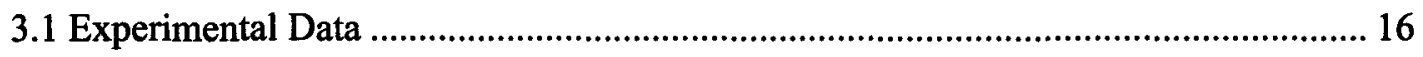

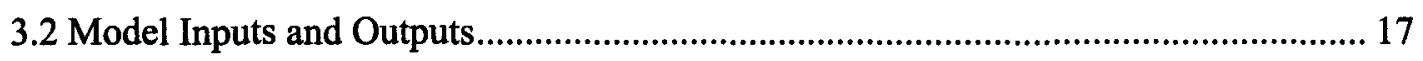

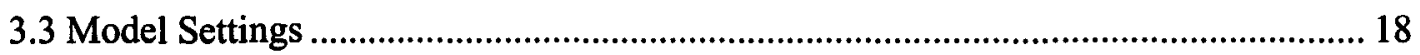

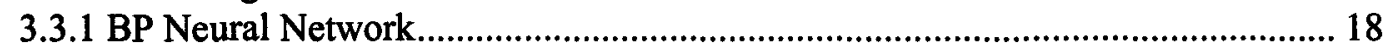

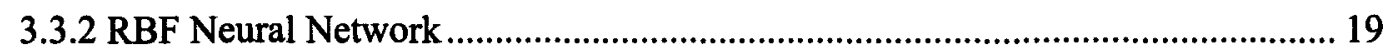

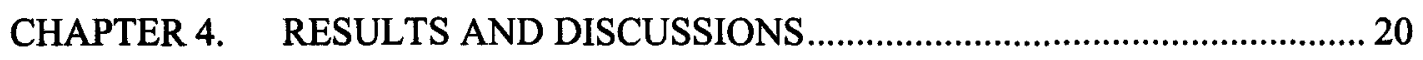

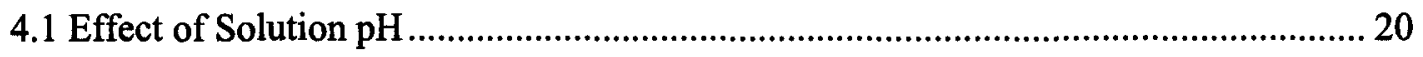

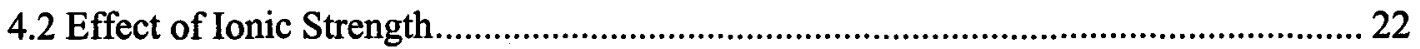




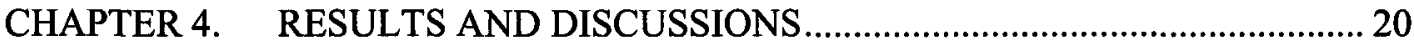

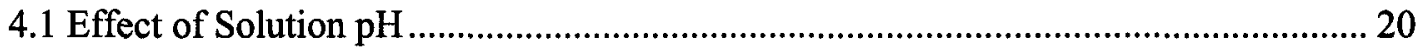

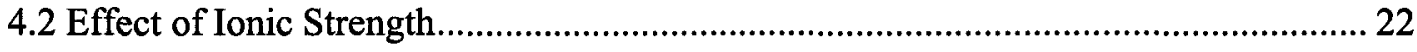

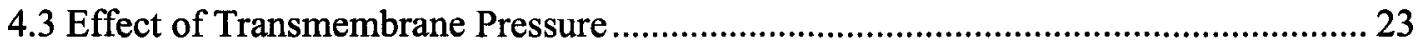

4.4 Performance of Multiple Regression, BPNN and RBFNN .................................. 23

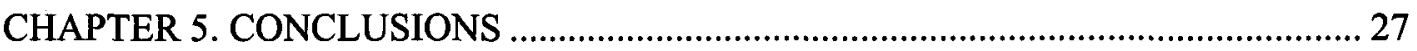

APPENDIX A. A SUPPLEMENTARY CASE STUDY ............................................ 40

APPENDIX B. EXPERIMENTAL DATA (TRAINING SET) .................................. 46

APPENDIX C. EXPERIMENTAL DATA (VALIDATION SET) ................................ 48

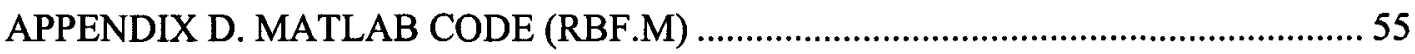

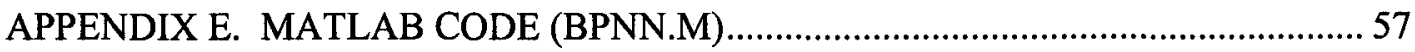

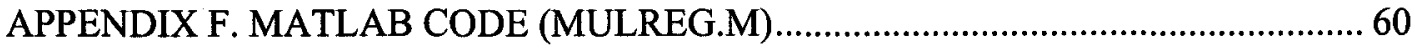

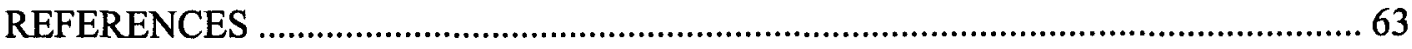




\section{LIST OF TABLES}

TABLE

$\underline{\text { PAGE }}$

1. Comparison of the performance of RBFNN, BPNN and multiple regression in terms of variance $\left(R^{2}\right)$, root mean square error (RMSE), number of points beyond $\pm 10 \%$ relative error $\left(N_{10}\right)$, and simulation time, given the same number of points for total data

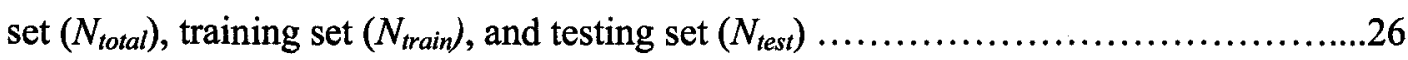




\section{LIST OF FIGURES}

FIGURE

$\underline{\text { PAGE }}$

1. Schematic diagrams of deadend and crossflow membrane filtrations .29

2. Architecture of BP neural network employed in this study. RBFNN inputs consist of particle size (PS), ionic strength (IS), solution $\mathrm{pH}$, transmembrane pressure $(\Delta \mathrm{P})$, and elapsed time $(\mathrm{t})$, which are used as input parameters to predict the permeate flux (J). $b_{1}$, is the bias in the input layer; $b_{2}$ and $b_{3}$ are biases in two hidden layers .30

3. Architecture of RBF neural network employed in this study. RBFNN inputs consist of particle size (PS), ionic strength (IS), solution $\mathrm{pH}$, transmembrane pressure $(\Delta \mathrm{P})$, and elapsed time $(\mathrm{t})$, which are used as input parameters to predict the permeate flux (J). $b_{1}$ and $b_{2}$ are biases in the input and hidden layer, respectively .31

4. A schematic diagram of the membrane test unit. (Adopted from Faibish et al. (1998))

5. Experimental data and ANN predictions on effect of solution $\mathrm{pH}$ on permeate flux decline with ionic strength of $10^{-1} \mathrm{M} \mathrm{KCl}$. The filtration conditions employed are 
particle size $47 \mathrm{~nm}$, transmembrane pressure $41.4 \mathrm{kPa}$ (6 psi), particle volume concentration $0.01 \%$, cross flow velocity $0.246 \mathrm{~m} / \mathrm{s}$, and temperature $20{ }^{\circ} \mathrm{C}$. At each $\mathrm{pH}$ value, the solid and the hollow symbols represent experimentally observed and ANN simulated data while $\times$ indicates data points used for the training process

6. Experimental data and ANN predictions on the effect of solution $\mathrm{pH}$ on permeate flux decline with ionic strength of $10^{-3} \mathrm{M} \mathrm{KCl}$. The filtration conditions employed are particle size $47 \mathrm{~nm}$, transmembrane pressure $41.4 \mathrm{kPa}$ (6 psi), particle volume concentration $0.01 \%$, cross flow velocity $0.246 \mathrm{~m} / \mathrm{s}$, and temperature $20^{\circ} \mathrm{C}$. At each $\mathrm{pH}$ value, the solid and the hollow symbols represent experimentally observed and ANN simulated data while $\times$ indicates data points used for the training process .34

7. Experimental data and ANN predictions on the effect of ionic strength on permeate flux decline for (a) $47 \mathrm{~nm}$, (b) $110 \mathrm{~nm}$ and (c) $310 \mathrm{~nm}$ particles. The filtration conditions employed were $\mathrm{pH} 10$, transmembrane pressure $41.4 \mathrm{kPa}$ (6 psi), particle volume concentration $0.01 \%$, cross flow velocity $0.246 \mathrm{~m} / \mathrm{s}$, and temperature $20{ }^{\circ} \mathrm{C}$. For each particle size, the symbol $\times$ and + indicate the selected data points of ionic strength of $10^{-4}$ and $10^{-1} \mathrm{M}$, respectively, for the training process. The solid symbols indicate experimentally observed data of ionic strength $10^{-4} M(\mathbf{\Delta}), 10^{-3} M(\boldsymbol{\square}), 10^{-2} M(\odot)$, and $10^{-1} M(\nabla)$ of $\mathrm{KCl}$ while the corresponding hollow symbols represent the simulation results .35 
8. Experimental data and ANN predictions on the effect of transmembrane pressure on permeate flux decline for ionic strengths of (a) $10^{-4} \mathrm{M}$, (b) $10^{-3} \mathrm{M}$ and (c) $10^{-2}$ $M \mathrm{KCl}$. The filtration conditions employed were $\mathrm{pH} 10$, particle size $47 \mathrm{~nm}$, particle volume concentration $0.01 \%$, cross flow velocity $0.246 \mathrm{~m} / \mathrm{s}$, and temperature $20^{\circ} \mathrm{C}$. For each particle size, the symbol $\times$ and + indicate the selected data points of transmembrane pressure of $62.1 \mathrm{kPa}(9 \mathrm{psi})$ and $20.7 \mathrm{kPa}$ (3 psi), respectively, for the training process. The solid symbols indicate experimentally observed data of transmembrane pressure $62.1 \mathrm{kPa}(9 \mathrm{psi})(\mathbf{\Delta})$, $41.4 \mathrm{kPa}(6 \mathrm{psi})(\mathrm{O})$, and $20.7 \mathrm{kPa}(3 \mathrm{psi})(\boldsymbol{\nabla})$ while the corresponding hollow symbols represent the simulation results .36

9. The comparison of a) RBFNN, b) BPNN and c) multiple regression predictions of permeate flux with experimental measurement under different operational conditions. The solid straight line represents the line of equality and the dashed lines represent $\pm 10 \%$ variance. The variance and RMSE between the predicted and measured data are also provided $.37 / 38 / 39$ 


\section{CHAPTER 1. INTRODUCTION}

Compared to conventional water and wastewater treatment systems, membrane separation is a more compact operation that provides higher quality products, easier operational control and less maintenance. The major aim for efficient membrane filtration is to minimize permeate flux decline (due to membrane fouling) caused by the accumulation of particles on the membrane surface and/or within pores during filtration processes. A plethora of studies on permeate flux decline indicate that particle accumulation during crossflow colloidal membrane filtration is affected by physical and chemical characteristics of the feed water as well as hydrodynamic operating conditions (Mcdonogh et al. 1989; Romero and Davis 1990; Bacchin et al. 1996; Faibish et al. 1998).

Design and application of membrane filtration require development of predictive models that can inter-link solution properties and operating conditions to filtration performance. To predict permeate flux decline, many theoretical models have been proposed using physical, chemical, and hydrodynamic modeling parameters such as particle size, zeta potential, Hamaker constant, transmembrane pressure, solution $\mathrm{pH}$, ionic strength, temperature, and so forth (Bowen and Jenner 1995). In most practical applications, effects of the concentration polarization (CP) layer are barely measurable since the cake layer is generated shortly after the CP layer forms. Thus, one of the main focuses of flux decline models is on the time-dependent changing of the cake layer 
resistance during filtration while the $\mathrm{CP}$ layer's effect is assumed to be negligible (Romero and Davis 1988; Romero and Davis 1990; Song and M 1995; Bacchin et al. 2002).

Film theory, gel layer, osmotic pressure, and boundary layer resistance are several of the theoretical models that have been developed (Bowen and Jenner 1995). However, the accuracy of theoretical modeling has been hampered by insufficient knowledge regarding the complexity of the micro-scale phenomena occurring during the filtration processes. In addition, theoretical model designs involve specific assumptions that lead to deviations between theoretical predictions and experimental observations. Such deviations imply that fundamental, theoretical models sometimes do not have sufficient capability to depict fouling phenomena occurring in real filtration systems although the models accurately depict fundamental transport mechanisms. Purely empirical models, which can be built upon specific experimental observations and used to predict the membrane system performance, appear to be new alternatives. The multiple regression is the simplest of all the multivariate statistical techniques and can find the linear relationship between one dependent variable (output) and many independent variables (inputs) (Wilks 1995). Another popular method is the Artificial Neural Network (ANN) approach, which can obtain either linear or nonlinear relationships between inputs and output(s).

The primary advantage of ANN over theoretical models is that, as a black box model, it does not pre-require any governing equation specifically describing the underlying engineering phenomena. Instead, ANN can learn the complex transport processes of a system from given observed data, serving as an instrument for universal 
data approximation. Accordingly, a considerable number of ANN applications for prediction, classification, signal processing and optimization can be found in various fields such as finance and economics, meteorology, chemistry and chemical engineering, computer science, water resources engineering, and so forth (Maas et al. 2000; Bowden et al. 2005; Bowden et al. 2005; Sahoo et al. 2005; Valverde Ramirez et al. 2005), exemplifying its great predictive potential of transport processes.

Among assorted neural networks, the multi-layer feed-forward neural network with backpropagation training algorithm, typically called backpropagation neural network (BPNN), may be one of the most widely used networks for information extraction and classification (Dornier et al. 1995; Niemi et al. 1995; Bowen et al. 1998; Chakraborty et al. 2003; Razavi et al. 2003; Shetty et al. 2003; Ramaswamy et al. 2004). However, BPNN exhibits several serious shortcomings, such as the slow convergence during its training step, susceptibility to converging to a local minimum, and inability to detect over-fitting (Schilling et al. 2001). For these aspects, the radial basis function neural network (RBFNN) seems a better alternative to BPNN as RBFNN provides easier initialization, faster training procedure and more stable performance.

Extensive research has been performed to investigate the capability of BPNN as a tool for membrane system modeling. Application of BPNN to membrane research is becoming a greatly researched topic because it has provided promising results. For example, BPNN has been harnessed to predict steady-state contaminant removal efficiency during nanofiltration in the drinking water treatment system (Shetty et al. 2003). BPNN's potential for dynamic simulation of membrane fouling during crossflow microfiltration has also been investigated (Dornier et al. 1995). Additional applications 
can be found in predicting permeate flux, hydraulic resistance and rejection for various types of feed solutions (Niemi et al. 1995; Bowen et al. 1998; Chakraborty et al. 2003; Razavi et al. 2003). Moreover, BPNN's ability for classification has been shown in the development of a phenomenological model to categorize membrane pore size using the ultrasonic signal amplitude (Ramaswamy et al. 2004).

To the best of our knowledge, any RBFNN application to membrane systems has not been reported yet. The goal of this study is to study an information-integrated RBFNN applied to membrane filtration analysis and to explore its predictability of the permeate flux under various operating conditions and solution characteristics. A brief introduction to multiple regression, BPNN, and RBFNN is given, and the effectiveness of RBFNN is compared to those of multiple regression and BPNN approaches. 


\section{CHAPTER 2. REVIEW OF LITERATURE}

\subsection{Membrane Filtration}

\subsubsection{Membrane Types By Pore Size}

Membrane filtration is one of the most widely used separation techniques. Depending on membrane porosity, the overall pressure-driven membrane process can be classified as microfiltration (MF), ultrafiltration (UF), nanofiltration (NF), and reversed osmosis (RO) in decreasing membrane pore size (Mallevialle et al. 1996; Mulder 1997). However, the underlying principles governing rejection of contaminant by the membrane substantially differ.

MF membranes, with pore sizes typically between $0.1 \mu \mathrm{m}$ and $10 \mu \mathrm{m}$, are generally used for clarification and removal of micro-particulates. Rejection of contaminants by the MF membranes is due entirely to physical sieving of the material at the membrane surface. UF and NF membranes may reject contaminants based on a combination of physical sieving and contaminant-membrane chemistries. UF membranes, with pore sizes between 0.001 and $0.1 \mu \mathrm{m}$, are used for concentrating and desalting macromolecules. NF (pore size between 1 and $10 \mathrm{~nm}$ ) and RO (pore size less than $1 \mathrm{~nm}$ ) membranes are typically used to remove low molecular weight solutes and salt ions, respectively. Rejection of contaminants by RO membranes largely depends on the differences in solubility and diffusivity (Mallevialle et al. 1996; Mulder 1997). 


\subsubsection{Membrane Filtration By Operation}

Based on the flow direction with respect to the membrane surface, membrane filtration processes can be generally classified into two categories: 1) deadend filtration, also known as "direct flow filtration", and 2) crossflow filtration, also known as "tangential flow filtration" (Fig. 1).

The feed flow direction of the deadend filtration is perpendicular to the membrane surface, and so $100 \%$ of feed fluid attempts to pass through the membrane, while suspended particles with sizes greater than that of the membrane pores are blocked. Deadend filtration is not of practical interest because of its rapid flux decline stemming from continuing particle deposition on the membrane surface. In contrast, crossflow filtration is commonly employed in large-scale, industrial membrane filtration because the feed solution flows parallel to the membrane surface; one portion passes through the membrane pores (permeate) while the remainder is disposed of and/or re-circulated to the feed reservoir. The tangential feed flow over the membrane surfaces enables suspended solids to be carried downstream using shear forces that significantly reduce particle accumulation on the membrane.

\subsubsection{Membrane Fouling}

The major aim for efficient membrane filtration is to minimize permeate flux decline (due to membrane fouling) caused by the accumulation of particles on the surface and/or within pores of the membrane during filtration processes. Fouling is an unavoidable, deleterious phenomenon in membrane filtration that causes serious 
permeate flux decline as well as deterioration of permeate quality. Particle deposition on the membrane surface can be typically classified into two processes: 1) concentration polarization (CP) and 2) cake layer formation between the CP layer and the membrane surface.

The solute concentration of the bulk phase is almost identical to that of the feed water (Fig. 1). . As solute (i.e. particles in this study) transporting from a bulk phase to and retained on the membrane surface, the solute concentration above the membrane surface is higher than that of the bulk phase. This layer is called the CP layer. The solute CP layer reaches its steady state after a short time period after the filtration process begins. Thereafter, suspended particles continuously accumulate on the membrane surface and form a dense cake layer which contributes to the major resistance of the permeate water flux (Mallevialle et al. 1996; Mulder 1997).

\subsubsection{Parameters}

Permeate flux (J) is defined as the volume of permeate per unit membrane surface per unit time (Mallevialle et al. 1996). The permeate flux from a membrane system decreases with time $(t)$, and eventually reaches a steady state, which is known as limiting flux. Various parameters can be modified to optimize the permeate flux during membrane filtration. In the following subsections, the definitions and effects of the four major physiochemical parameters are explained. 


\subsubsection{Transmembrane Pressure $(\Delta P)$}

Transmembrane pressure is the driving force of membrane separation, defined as the pressure difference between the filtrate and the permeate sides of the membrane (Kim 2004). In general, an increase in the transmembrane pressure monotonously enhances the flux across the membrane. The exception to this generalization occurs if a compressible cake layer is present on the surface of the membrane, where the increased transmembrane pressure can compress the pre-built cake layer, leading only to a slight increase in the permeate flux.

\subsubsection{Solution pH}

Solution $\mathrm{pH}$ value is an indicator of the molar concentration of hydrogen ions in solution (Mulder 1997; Kim 2004). In general, a lower permeate flux is associated with low solution $\mathrm{pH}$ due to the decrease in the apparent viscosity of permeating solution near the negatively-charged membrane pores. The influence of membrane pores on the liquid viscosity can be explained by the so-called electroviscous effect, which is less pronounced at higher ionic strength or near capillary surface of zero charge. At high ionic strength or low surface charge, the solution appears to exhibit a reduced viscosity when compared with the flow at low ionic strength and high surface charge.

\subsubsection{Ionic Strength (IS)}

Ionic strength is a measure of the composite effect of all ions in the feed solution, defined as half of the total sum of the concentration of all the ionic species in the solution multiplied by the square of their charge. The inter-particle distance in the cake layer 
decreases at higher ionic strengths due to the decrease in the electrostatic double layer repulsive force. Consequently, a solid-packed cake layer promotes the resistance to permeate flux (Mulder 1997).

\subsubsection{Particle Size (PS)}

The suspended particle size in our study is represented by particle diameter. When larger particles are used to make the cake layer, they tend to pack with larger pores among adjacent particles, providing easier pathways for water to penetrate the layer. Changes in particle size obviously affect interparticle interactions due to more repulsive or attractive pair-wise forces, depending on the zeta potential and Hamker constant of particles, as well as ionic strength of the feed solution. However, variation in volume faction of a cake layer due to particles size changes would not be more important than actual pore size increase, which contributes significantly less hydraulic resistance (Kim 2004).

\subsection{Methodologies}

\subsubsection{Multiple Regression}

Using a multiple regression is the simplest method to obtain the relationship between a continuous output $(\hat{y})$ and several inputs (Wilks 1995). Mathematically, multiple regression is a straightforward generalization of simple linear regression. There are $k$ independent inputs, $x_{1}, x_{2}, x_{3}$ and so on up to $x_{k}$. Predicted values from multiple regression are linear combinations of the predictor variables. Therefore, the general form of a prediction equation from the multiple regression is 


$$
\hat{y}=a_{0}+a_{1} x_{1}+a_{2} x_{2}+\ldots+a_{k} x_{k}
$$

where $a_{1}$ is the coefficient of $x_{1}, a_{2}$ is the coefficient of $x_{2}$, and so forth. The coefficient of each independent variable indicates what relationship that variable has with the dependent variable $\hat{y}$, when all the other independent variables are held constant. Multiple regression solves for unknown coefficients $a_{0}, a_{1}, a_{2}, \ldots$, to $a_{k}$ by performing a least squares fit, which generally aims to minimize the sum of the squared deviation of the observed values given the dependent variable from those predicted by the model. The least square estimator (LSE) has the following form:

$$
L S E=\sum_{i=1}^{n}\left(y_{i}-\hat{y}\right)^{2}
$$

where $y$ is the observed dependent variable.

\subsubsection{Artificial Neural Network}

\subsubsection{Introduction of ANN}

Artificial Neural Network (ANN) is a parallel-distributed information-processing system (ASCE 2000) with a large number of neurons and synapses (inter-neuron connections), which is inspired by biological systems such as the human brain. It is estimated that the human brain contains over 100 billion neurons and $10^{14}$ inter-neuron connections. Neurons and synapses constitute the key elements for neural information processing, forming a neural network. Scientists construct a numerical model, called an artificial neural network, based on the structure and behavior of the biological neural network.

How neurons interact is different for different neurons. Generally speaking, a neuron sends its output to other neurons. Neurons' synapses collect signals by summing 
all the excitatory and inhibitory influences acting on it, which is represented by a simple threshold function known as transfer function or activation function in an ANN model.

As a numerical model, the uniqueness of ANN lies in its ability to learn and generate interrelationships between the input and output of observed or experimental data without requiring any postulates and assumptions. ANNs can be classified into different categories by their network architecture, activation or transfer function, and training algorithm (Fausett 1994). A neural network's architecture defines the number of neurons and layers within the network. Training is the learning process by which an ANN calculates the interrelationship between inputs and output(s) from examples, and an optimal training algorithm could speed up the training procedure.

Generally, neurons in ANN are arranged in input, hidden and output layers. The neurons are then linked to others with associated weights and biases, which will be adjusted to optimal values during the training. The Neural Network Toolbox available in MATLAB (Mathworks, Inc., Natwick, MA) (Demuth and Beale 2003) was used to design, train and simulate the BPNN and RBFNN in this study. Later, the two neural networks are compared in terms of their performance.

\subsubsection{Backpropagation Neural Network}

The multiplayer feed-forward BPNN is one of the most widely employed ANN models in membrane study. The term "backpropagation" refers to the way in which the gradient is computed for nonlinear multiplayer networks. The BPNN usually can have multiple hidden layers, besides the input and output layers (Fig. 2). The input and output layers serve as nodes to buffer input and output for the model, respectively. Hidden 
layers provide a means for relating input and output. Before running the model, weights for the neurons are randomly assigned, which makes the network much like a newborn's brain, developed but without knowledge. When training the network, once the model prediction is given, it is compared to the actual data. The adjustment based on the error between the predicted and measured data is then "backpropagated" through the network, which causes the weight adjustment. The modification of weights is done by pointing the error to the local minimum. Unfortunately, the local minimum is not always the global minimum, which will cause the model to settle in an non-optimal configuration. This is the major weakness of BPNN. More introductory overviews of BPNN can be found in several studies (Dornier et al. 1995; Niemi et al. 1995; Bowen et al. 1998; Chakraborty et al. 2003; Razavi et al. 2003; Shetty et al. 2003; Ramaswamy et al. 2004).

A transfer function is the equation computing the output of a node in a hidden or output layer. The tan-sigmoid function is the transfer function employed in the hidden layers in this study and has the following form:

$$
f(n)=\tan \left(\frac{1}{1+e^{-n}}\right)
$$

where $n$ is the net input to the neuron, which is the sum of the products of connection weight and output value from connected neurons of the previous layer with the associated bias. This transfer function is bounded between -1 and 1 so input data are usually normalized to the same range. Neurons in the output layer receive weighted input and generate the final output using a linear transfer function:

$$
\hat{y}=\sum_{i=1}^{m} w_{i} f_{i}(n)+b_{3}
$$


where $\hat{y}$ is the BPNN simulation result, $w_{i}$ is the optimized connection weight determined through the training process (ASCE 2000), and $b_{3}$ is the bias in the output layer.

Training is an optimization procedure in which the network weights are adjusted in order to minimize the selected error value. The error function is function that measures the difference between the target and model predicted output, as well as the generalization ability of models. Both the mean square error (MSE) and root mean square error (RMSE) were the commonly used functions to estimate the performance of the neural network:

$$
\begin{aligned}
M S E & =\frac{1}{n} \sum_{i=1}^{n}\left(y_{i}-\hat{y}_{i}\right)^{2} \\
R M S E & =\sqrt{\frac{1}{n} \sum_{i=1}^{n}\left(y_{i}-\hat{y}_{i}\right)^{2}}
\end{aligned}
$$

where $y$ is the target output value (or observed data), $\hat{y}$ is the network simulation output, and $n$ is the total numbers of data patterns (input and output pairs) used.

\subsubsection{Radial Basis Function Neural Network}

RBFNN is another type of neural network. Such networks generally have 3 layers, the input layer, the hidden layer with the RBF non-linearity, and the linear output layer. RBFNN can overcome some of the limitations of BPNN by using a rapid training phase, having a simple architecture, and maintaining complicated mapping abilities.

In most cases, the RBFNN simulates an aimed phenomenon by using Gaussian basis functions in the hidden layer and linear transfer functions in the output layer. The 
major difference in operation between the RBFNN and BPNN exists in the hidden layer. Instead of the weighted sum of the input vector used in BPNN, the distance between the input and center, as explained below, is used in the RBFNN learning process.

The first layer of the RBFNN collects the input data. Its training process determines the number of hidden neurons, $m$, which can be larger than that of BPNN to achieve a certain accuracy of prediction (Demuth and Beale 2003). For each neuron in the hidden layer, the distance between the input data and the center is activated by a nonlinear radial basis function, as shown in the following equation:

$$
R_{i}=\exp \left[-\left(\left\|x_{i}-c_{i}\right\| b_{1}\right)^{2}\right]
$$

where $x_{i}$ is the input vector, and $b_{1}$ and $c_{i}$ are parameters that represent the bias in the hidden layer and center vector, respectively. Each neuron in the hidden layer will produce a value between 0 and 1 according to how close the input is to the location of the center. Therefore, neurons with centers closer to inputs will have more contributions to outputs; however, if neurons have centers far away from inputs, then the outputs would be nullified. Later, the output layer neurons receive the weighted inputs from the hidden layer and produce the results by using a linear combination, which is of a similar form to that of the BPNN:

$$
\hat{y}=\sum_{i=1}^{m} w_{i} R_{i}+b_{2}
$$

where $\hat{y}$ is the RBFNN simulation result, $w_{i}$ is the optimized connection weight determined through the training process (ASCE 2000), and $b_{2}$ is the bias in the output layer. 
Figure 3 gives an insight into the structure and working procedure of the RBFNN. The open circles are bias neurons that adjust the sensitivity of the network. The biases are controlled by a specific value, called "spread" number, and each bias is set to 0.8326/spread (Demuth and Beale 2003). The selected "spread" value should be large enough for neurons in the hidden layer to include the whole range of input data.

In RBFNN simulations, the proper initial choice of centers and weights should be regarded as key issues. Various methods are proposed to define the center, e.g. random selection, K-mean technique, max-min algorithms, etc. (Alldrin et al. 2004) Using the Neural Network Toolbox, we chose the weight vectors as the center parameter. Instead of being randomly generated, the initial value of weights was set as the transpose of the input vector according to the MATLAB Toolbox (Demuth and Beale 2003). Once the centers have been developed, the weights linking the hidden and output layers should be updated during the training procedure. The training procedure in the RBFNN also determines the number of hidden neurons required for the simulation. The training of RBFNN is initiated by first generating a single neuron in the hidden layer, followed by continuously adding neurons to the hidden layer one at a time. 


\section{CHAPTER 3. DATA AND MODEL SETTINGS}

\subsection{Experimental Data}

The experimental data used in this study were adopted from a paper published by Faibish et al. (1999) [1]. Three different silica $\left(\mathrm{SiO}_{2}\right)$ particles were selected as suspended colloids for the membrane filtration experiments. These three different types of silica particles, denoted as ST-XL, PST-1, and PST-3 produced by the manufacturer (Nissan Chemicals, Tarrytown, NY), have mean particle diameters of 47, 110 and 310 $\mathrm{nm}$, respectively. A negatively charged UF membrane with pore size equal to $20 \mathrm{~nm}$ was employed in the laboratory-scale membrane test; hence, complete rejection of suspended solids on the membrane surface was observed. The schematic diagram in Figure 4 shows the membrane test unit used in these experiments. The colloidal suspension was held in a polypropylene reservoir and fed to the inlet port of the membrane module by a peristaltic pump. The feed flow rate of suspension was measured by a flow meter connected between the pump outlet and membrane inlet. A variable reluctance differential pressure transducer measured the transmembrane pressure, controlled by a needle valve installed on the outlet side of the membrane module. By weighing permeate collected in a pre-weighted beaker, the permeate flux could be measured. 


\subsection{Model Inputs and Outputs}

In the experimental study, the effects of solution $\mathrm{pH}$, ionic strength (IS), and transmembrane pressure $(\Delta P)$ on the permeate flux $(J)$ in crossflow membrane filtration were examined. In order to examine the effect of a particular parameter in each experiment, other parameters were fixed and the permeate flux was measured accordingly. Various other operational parameters such as particle bulk concentration, temperature, and crossflow velocity were involved. In order to reduce the complexity and improve performance of the network simulation, however, the least number of inputs were selected: particle size (PS), ionic strength (IS), solution $\mathrm{pH}$, transmembrane pressure $(\Delta P)$, and elapsed time $(t)$ of the filtration process (Appendix B and C). The IS value was expressed in logarithmic scale in the simulation due to its incongruous value (wide range of data value). The multiple regression, BPNN and RBFNN were constructed to predict the variation of permeate flux with respect to time under the above physical, chemical and hydrodynamic operation parameters.

A total of 567 data points were collected from the experiments. In order to use the minimum training set in the study, the minimum ratio of training to verification data set with satisfying results are listed, according to a trial-and-error method. Approximately $17 \%$ (96 data points) of all the experimental data were used for training (Appendix B) and obtaining the interrelationship between inputs and outputs, and the remaining $83 \%$ (471 data points) were used for model performance verification (Appendix C). As all empirical models are only able to interpolate between the application boundary conditions (Shetty et al. 2003), the training data should be 
representative of the entire range of experimental conditions. Therefore, extreme values of the experimental data need to be included as part of the training set.

\subsection{Model Settings}

\subsubsection{BP Neural Network}

Among many BPNN training methods available in the MATLAB Toolbox, the Levenberg-Marquardt training algorithm was selected because of its rapid convergence rate (Demuth and Beale 2003).

Learning rate is the option to regulate the training speed. It indicates the rate of change of connection weights during training. The selection of a learning rate is of critical importance in finding the minimum error. The typical learning rate is greater than 0 and less than 1 . Lower learning rates require more training iterations. While a higher learning rate allows the network to converge more rapidly, the chances of a non-optimal solution are greater. To ensure the optimal performance of BPNN, the learning rate in this study was set to 0.01 .

Another option in controlling the training procedure is the number of epochs, which represents the total number of times the entire set of training data will pass through the network structure. Increasing this number will likely improve the accuracy of the model, but at the cost of time. Conversely, decreasing the number of epochs will likely lower the accuracy, but take less time. A moderately high number of epochs, 400 , was assigned for this study.

During the backpropagation training, the validation of suitability of the neural network simulation was estimated by calculating the Mean Square Error (MSE) between 
the observed and ANN-simulated data. In our study, the maximum number of epochs, target error goal 'MSE', and minimum performance gradient were set to $400,10^{-10}$, and $10^{-6}$, respectively. Training stops when the maximum number of epochs was reached, or either the MSE or performance gradient were minimized to arrive at the pre-determined goal. Through a trial-and-error method, the optimal network structure for the current BPNN simulation was determined to have two hidden layers, one containing 4 and the other containing 2 hidden neurons as shown in Figure 2.

\subsubsection{RBF Neural Network}

The model setting of RBFNN is relatively easier than BPNN. Before training starts, only a few key training parameters should be assigned. In our study, the target error goal MSE and the maximum number of neurons were set to $10^{-10}$ and 80 , respectively. Training was stopped when either of the following criteria was met: 1) MSE was minimized to be less than the pre-determined goal; 2) the maximum number of neurons was reached. Because the value of spread affects the quality of prediction, a trial-and-error method was employed, wherein several spread numbers are tested and the best one is accepted. By doing so, we found that the optimal spread number was 80 . 


\section{CHAPTER 4. RESULTS AND DISCUSSIONS}

Changing the physical, chemical and operating conditions is a useful technique to determine their effects on permeate flux. Experimental data, according to Faibish et al.'s paper, are divided into three sets to represent different parameters' effects on permeate flux. To ensure that the trained RBFNN can be used for prediction under various conditions, sets of six training points were selected from permeate samples in those three data sets as shown in Figs 5-8 (Appendix B). In this section, our model simulation results are presented and compared to Faibish et al.'s experimental observation.

\subsection{Effect of Solution pH}

In order to study the effects of $\mathrm{pH}$ on the permeate flux, the solution $\mathrm{pH}$ value was regulated by adding $\mathrm{NaHCO}_{3}$ or $\mathrm{NaOH}$ to the feed solution (Faibish et al. 1998). Figures 5 and 6 show comparisons of the RBFNN predictions and the experimentally observed permeate fluxes at two $\mathrm{pH}$ values of the feed solution $\left(10^{-1}\right.$ and $\left.10^{-3} \mathrm{MKCl}\right)$. Intermittent training data, shown as $\times$, were selected from the permeate flux profile of $\mathrm{pH} 6.1$ and 10.0 and then used to predict all the flux decline behaviors at pHs of $6.1,8.3$ and 10.0. The results generally depict the excellent performance of the RBF network as it accurately interpolates and reproduces the complex non-linear profiles of the transient permeate flux decline under various solution $\mathrm{pHs}$ and ionic strengths. 
The correlation coefficient between the observed and predicted permeate flux was as high as 0.993 , although minor departures were observed during the early stages of filtration, where permeate flux degenerates dramatically. The paired t-tests indicated that there was no statistical difference between the experimental values and neural network predictions at the $99 \%$ confidence level for both high $\left(10^{-1} \mathrm{M}\right)$ and low $\left(10^{-3} \mathrm{M}\right)$ ionic strengths.

Both model prediction and experimental data demonstrated that the feed water $\mathrm{pH}$ had only minor effects on the permeate flux. Comparing Figures 5 and 6 indicated that the initial permeate flux slightly decreased with $\mathrm{pH}$ and this behavior was captured by the RBFNN. However, the trained RBFNN did not accurately duplicate the almost identical permeate fluxes at pHs 8.3 and 10.0 in Figure 6. The neural network slightly overpredicted the initial permeate flux at $\mathrm{pH} 8.3$ for the first 10 minutes of filtration. We think this over-prediction stemmed from preparing the training data set only from $\mathrm{pH} 6.1$ and 10.0 cases, excluding $\mathrm{pH} 8.3$ data.

Comparison of Figures 5 and 6 showed that initial permeate flux increases at higher ionic strength and lower $\mathrm{pH}$ due to the narrow capillary effect on fluid viscosity. At the initial stage, when particle deposition was negligible, the intrinsic membrane resistance dominated. Under low $\mathrm{pH}$ and high ionic strength conditions, the decreasing fluid viscosity near membrane pores allowed water to penetrate the membrane pores with relative ease, leading to the initial enhanced permeate flux. However, at the later stages of the membrane filtration when the cake resistance became dominant, the steady state permeate fluxes became simply independent of the solution $\mathrm{pH}$. 


\subsection{Effect of Ionic Strength}

The RBFNN's predictability of the influence of ionic strength on the permeate flux was investigated using three difference particle sizes of $47 \mathrm{~nm}, 110 \mathrm{~nm}$ and $310 \mathrm{~nm}$, and four ionic strength values of $10^{-4}, 10^{-3}, 10^{-2}$, and $10^{-1} \mathrm{MKCl}$. The experimental data and the modeling results are shown in Figure 7, which clearly revealed the excellent predictability of the RBFNN for permeate flux over a wide range of ionic strengths and particle sizes. The predicted and experimental data did not show noticeable difference with correlation coefficient of 0.9944 and RMSE of 0.076 at the $99 \%$ confidence level through the entire time-dependent permeate flux.

As expected, increasing ionic strength promoted severe permeate flux decline and accelerated reaching a limiting flux level because the electrostatic double layer repulsion was mollified by the increasing ionic strength, causing a dense cake layer of enhanced hydraulic resistance. In Figures $7(a)$ and $7(b)$, the RBFNN closely captured the underlying fundamental effects of the double layer repulsion through various ionic strength on the permeate flux. On the other hand, Figure $7(\mathrm{c})$ reveals a quite different trend of transient fluxes from those of Figure 7(a) and 7(b), which seem to converge into a single limiting permeate flux level. This behavior indicated that the ratio of cake resistance to particle size can suppress the influence of interparticle interactions, leaving hydrodynamics as a dominating mechanism of flux decline. The trained RBFNN shows its ability to predict flux decline for ionic strengths of $10^{-2}$ and $10^{-3} \mathrm{M}$ by learning two different types of underlying mechanisms, i.e. interparticle and hydrodynamic interactions. 


\subsection{Effect of Transmembrane Pressure}

The ability of RBFNN to simulate the transmembrane pressure effect on the permeate flux is examined in this section. Results shown in Figure 8 demonstrate that RBF model can closely predict the permeate flux under different transmembrane pressures of $62.1,41.4$, and $20.7 \mathrm{kPa}$. The accuracy of the prediction is reflected by a correlation coefficient of 0.994 and RMSE of 0.092 .

Figure 8 shows that the permeate flux tends to reach a converging limiting flux under the same ionic strength regardless of applied transmembrane pressure and initial flux. As expected, Figure 8 confirmed that flux decline was accelerated by increasing transmembrane pressure and ionic strength (as described in section 4.2). A higher transmembrane pressure elevated the initial permeate flux by pushing more water toward a clean membrane while the particle concentration polarization was still developing. Contrary to the gain in the initial stage, the higher transmembrane pressure at a later stage pulled more particles toward the membrane surface by providing faster permeate velocity and thus contributed to the rapid generation of the deposit cake layer which caused dramatic decline of the permeate flux. Moreover, the higher pressure significantly compressed the pre-built cake layer so that it became denser and additionally contributed to the rapid flux decline. Decreasing flux is observed when both the initial flux and ionic strength are low which reflects slow and sparse formation of the cake layer.

\subsection{Performance of Multiple Regression, BPNN and RBFNN}

As noted in the introduction, BPNN has been widely used in the past decade as the standard ANN method to model membrane performance (Dornier et al. 1995; Niemi 
et al. 1995; Bowen et al. 1998; Chakraborty et al. 2003; Razavi et al. 2003; Shetty et al. 2003; Ramaswamy et al. 2004). This section mainly describes the superiority of RBFNN over BPNN by comparing their performances using $96\left(=N_{\text {train }}\right)$ filtration data points for training and $471\left(=N_{\text {test }}\right)$ points for testing out of total $567\left(=N_{\text {total }}\right)$ points of permeate flux vs. filtration time.

Figure 9(a) summarizes the RBFNN predictions for various operational conditions in Figures 5 to 8 by plotting predicted values as a function of experimental values of identical input parameters. The solid line in Figure 9(a) represents the perfect 1:1 match of the measured and predicted values of the permeate flux, and the dotted lines depict $\pm 10 \%$ relative error from the 1:1 match line. Most of data shown in Figure 9(a) are located near the $1: 1$ match straight line. The number of points beyond $\pm 10 \%$ relative error lines, $N_{10}$ is 14 when the number of training data set $N_{t e s t}$ is 471 , indicating $97 \%$ of the points are located within the $\pm 10 \%$ relative error. This excellent performance of RBFNN is also quantified by a RMSE of 0.082 and the variance $\left(R^{2}\right)$ of 0.988 . In spite of broad variations of feed water parameters and operational conditions, which cause complexity to the filtration system, the results overall indicate that employing only $17 \%$ (96 out of 567 points) of the total data for training renders satisfactory prediction of the remaining $83 \%$ ( 471 out of 567 points) of the data.

Previous investigations have suggested that RBFNNs have multiple advantages in comparison with the performance of BPNNs. In order to verify this concept, an undifferentiated simulation was also carried out by BPNN, and the result is presented in Figure 9(b). The hollow circles are clouded near the 1:1 match line, suggesting that BPNN is also able to accurately predict the permeate flux under different operational 
status to a certain extent. However, when compared with the RBFNN prediction in Figure 9(a), BPNN produces a somehow more scattered plot compared to that of the RBFNN, and $61\left(=N_{10}\right)$ out of 471 points are not confined within the two dotted lines. (Note that $N_{10}$ of the RBFNN is 14. ) Only $87 \%$ of the predicted points are within acceptable error whereas $97 \%$ were within $\pm 10 \%$ relative error using RBFNN.

The multiple regression equation obtained from the training data has the following form:

$$
\hat{J}=0.943+0.025[\Delta P]+0.1663[I S]-0.0192[p H]+0.0028[P S]-0.0133[t]
$$

A regression equation can also be used to predict values of the permeate flux for the remaining data. Results from multiple regression are additionally shown in Figure 9(c). It is clearly evident that, given the same information, multiple regression has a less accurate performance, mainly because the complex nonlinear membrane filtration process could not be presented by simple linear regression. Compared with Figure 9(a) and 9(b), hollow circles in Figure 9(c) are more detached from the 1:1 match line. More than half $(51.6 \%)$ of the predicted points $(471)$ were out of the $\pm 10 \%$ relative error range.

Finally, Table 1 compares the performance of RBFNN, BPNN and multiple regression using four parameters - variance $\left(R^{2}\right)$, RMSE, $N_{10}$ and required simulation time. RBFNN much more closely predicts the experimental observations in comparison to BPNN and multiple regression. Additionally, the RBF training method eventually speeds up the training process of the neural network by as much as 40 times over the BPNN training rate, providing higher adaptability when a large set of observed data is used to investigate more complex filtration systems. Nevertheless, comparing the model result for one data set alone cannot prove the robust advantage of RBFNN over the permeate 
flux prediction. For this reason, an supplementary case study was conducted in Appendix A to serve as the additional support of the superiority of RBFNN over BPNN and multiple regression on membrane permeate flux prediction.

Table 1. Comparison of the performance of RBFNN, BPNN and multiple regression in terms of variance $\left(R^{2}\right)$, root mean square error (RMSE), number of points beyond $\pm 10 \%$ relative error $\left(N_{10}\right)$, and simulation time, given the same number of points for total data set $\left(N_{\text {total }}\right)$, training set $\left(N_{\text {train }}\right)$, and testing set $\left(N_{\text {test }}\right)$.

\begin{tabular}{c|c|c|c|c|c|c|c}
\hline & $N_{\text {total }}$ & $N_{\text {train }}$ & $N_{\text {test }}$ & $R^{2}$ & RMSE & $N_{l 0}$ & $\begin{array}{c}\text { Simulation } \\
\text { time (sec) }\end{array}$ \\
\hline RBFNN & 567 & 96 & 471 & 0.988 & 0.082 & 14 & 2.47 \\
\hline BPNN & 567 & 96 & 471 & 0.958 & 0.156 & 61 & 96.32 \\
\hline MR & 567 & 96 & 471 & 0.755 & 0.332 & 244 & 0.046 \\
\hline
\end{tabular}




\section{CHAPTER 5. CONCLUSIONS}

Due to the complex physicochemical micro-phenomena occurring during membrane filtration, conventional theoretical models have been able to predict the filtration procedure only under limited conditions and/or with specific and/or inevitable assumptions. An artificial neural network method provides a unified approach for analysis and prediction of the performance of membranes under various combinations of operational conditions. In this study, the RBF neural network was applied to predict the permeate flux on crossflow membrane filtration as a function of transmembrane pressure, ionic strength, solution $\mathrm{pH}$, particle size and elapsed filtration time. Results show that the transient profiles of the permeate flux during the crossflow membrane filtration can be predicted by a single RBFNN with acute accuracy given a limited number of training data. Comparison of the performances of RBFNN, BPNN and multiple regression confirmed the superiority of the RBFNN in terms of the variance, RMSE, $N_{10}$ and simulation time required of higher accuracy.

As a consequence, it may not be necessary to carry out an entire series of expensive pilot or full-scale tests to collect and verify filtration data. The RBFNN can interpolate the performance of membrane filtration under other conditions of interest by using widely ranged but sparse data points to reduce the time and costs required. Although pilot scale experimental tests restrict the number of operating parameters that 
generally are of importance in larger scale applications, they still can closely simulate full-scale operations. In the same manner, RBFNN can also serve as potential prediction tools for full-scale operation, possibly combining several trained networks of different scales into one network. 

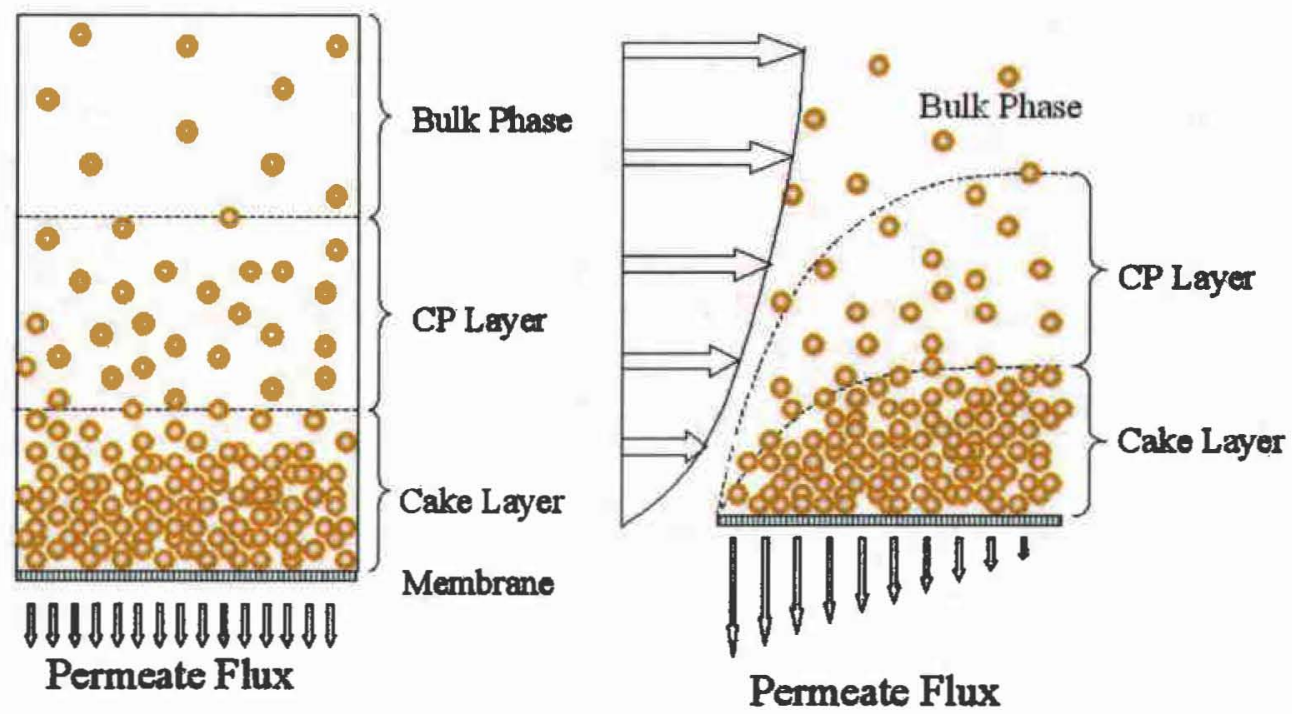

Figure 1. Schematic diagrams of deadend and crossflow membrane filtrations. 


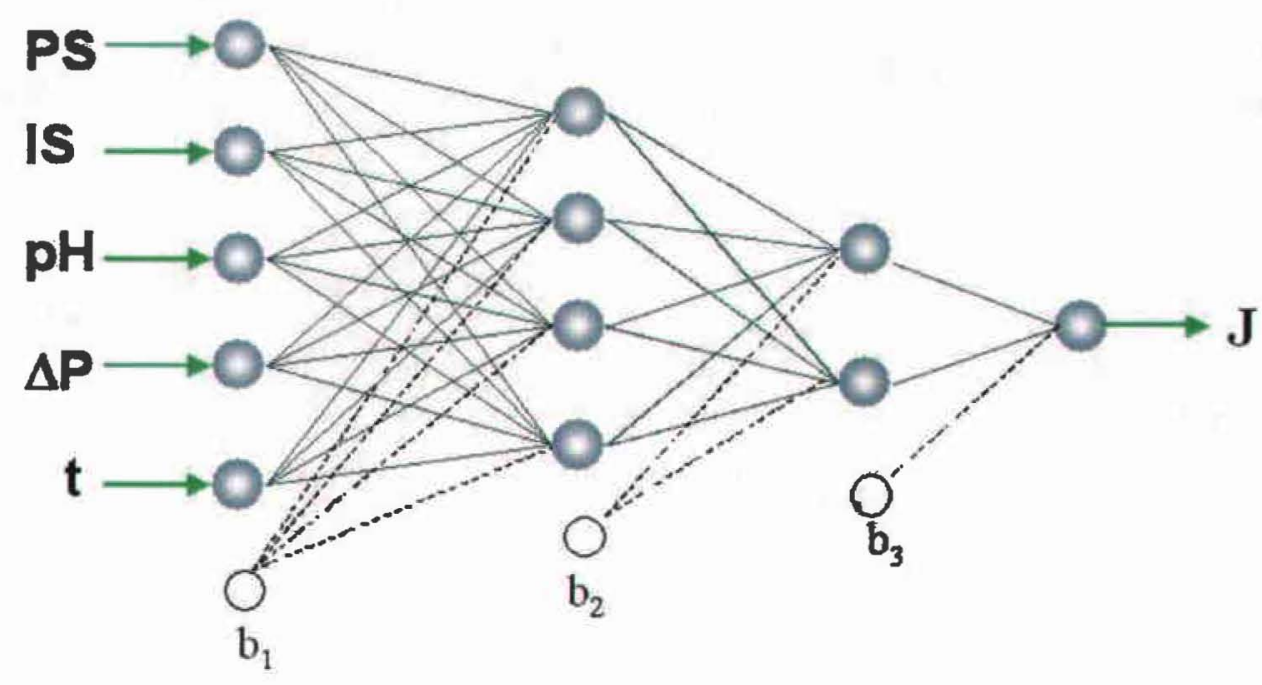

Figure 2. Architecture of BP neural network employed in this study. BPNN inputs consist of particle size (PS), ionic strength (IS), solution $\mathrm{pH}$, transmembrane pressure $(\Delta P)$, and elapsed time $(t)$, which are used as input parameters to predict the permeate flux (J). $b_{1}$, is the bias in the input layer; $b_{2}$ and $b_{3}$ are biases in two hidden layers. 


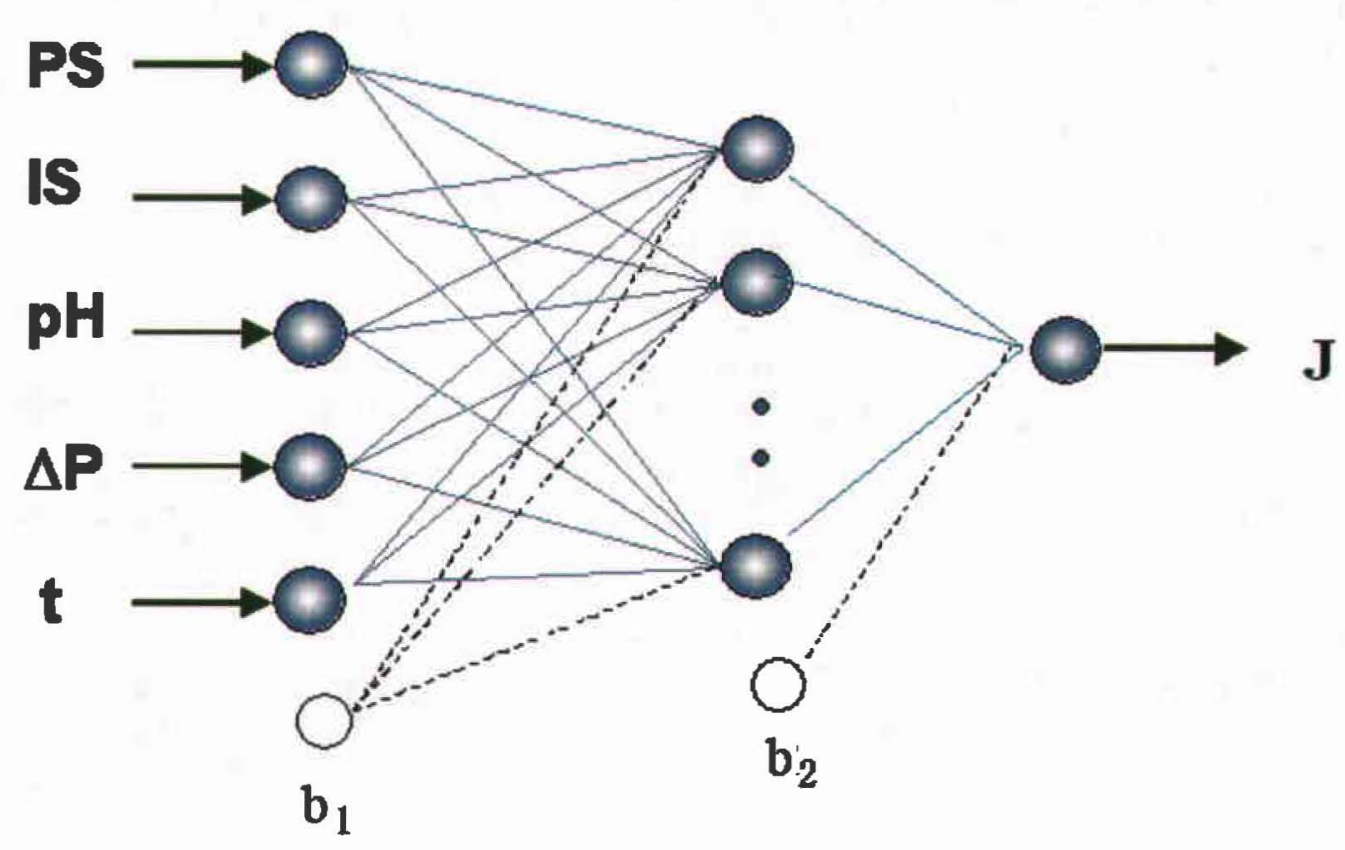

Figure 3. Architecture of RBF neural network employed in this study. RBFNN inputs consist of particle size (PS), ionic strength (IS), solution $\mathrm{pH}$, transmembrane pressure $(\Delta P)$, and elapsed time $(t)$, which are used as input parameters to predict the permeate flux (J). $b_{1}$ and $b_{2}$ are biases in the input and hidden layer, respectively. 


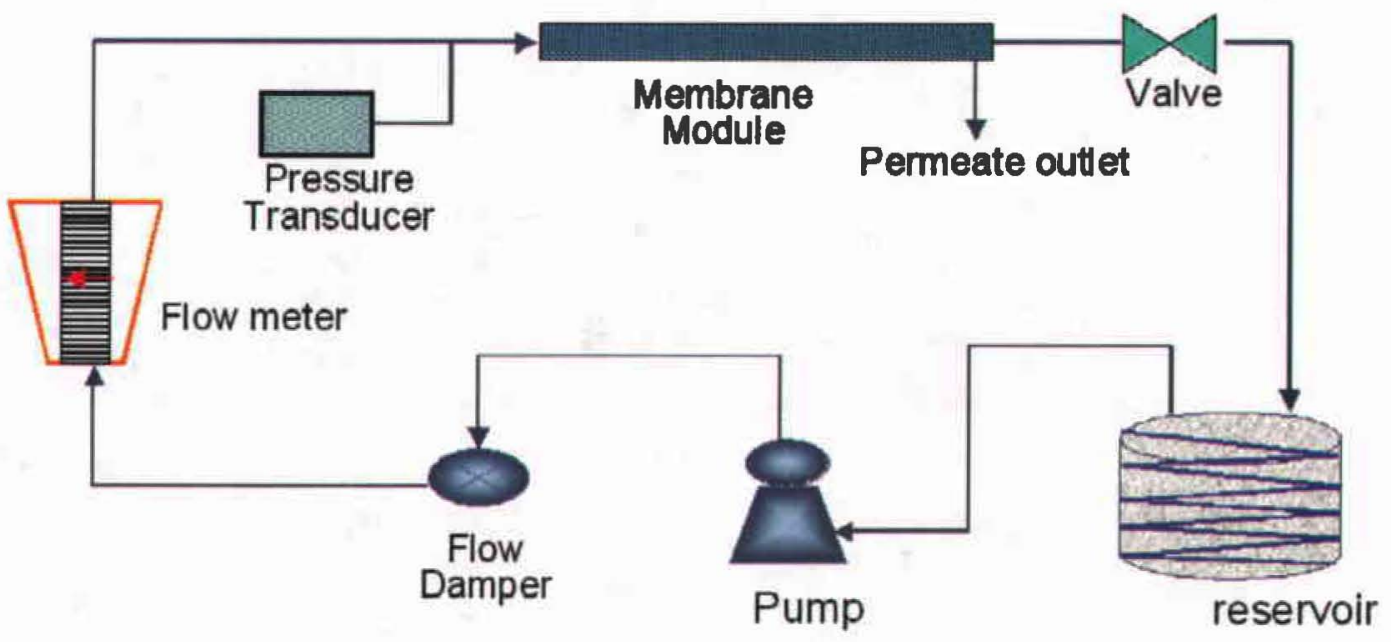

Figure 4. A schematic diagram of a membrane test unit. (Adopted from Faibish et al. (1998)). 


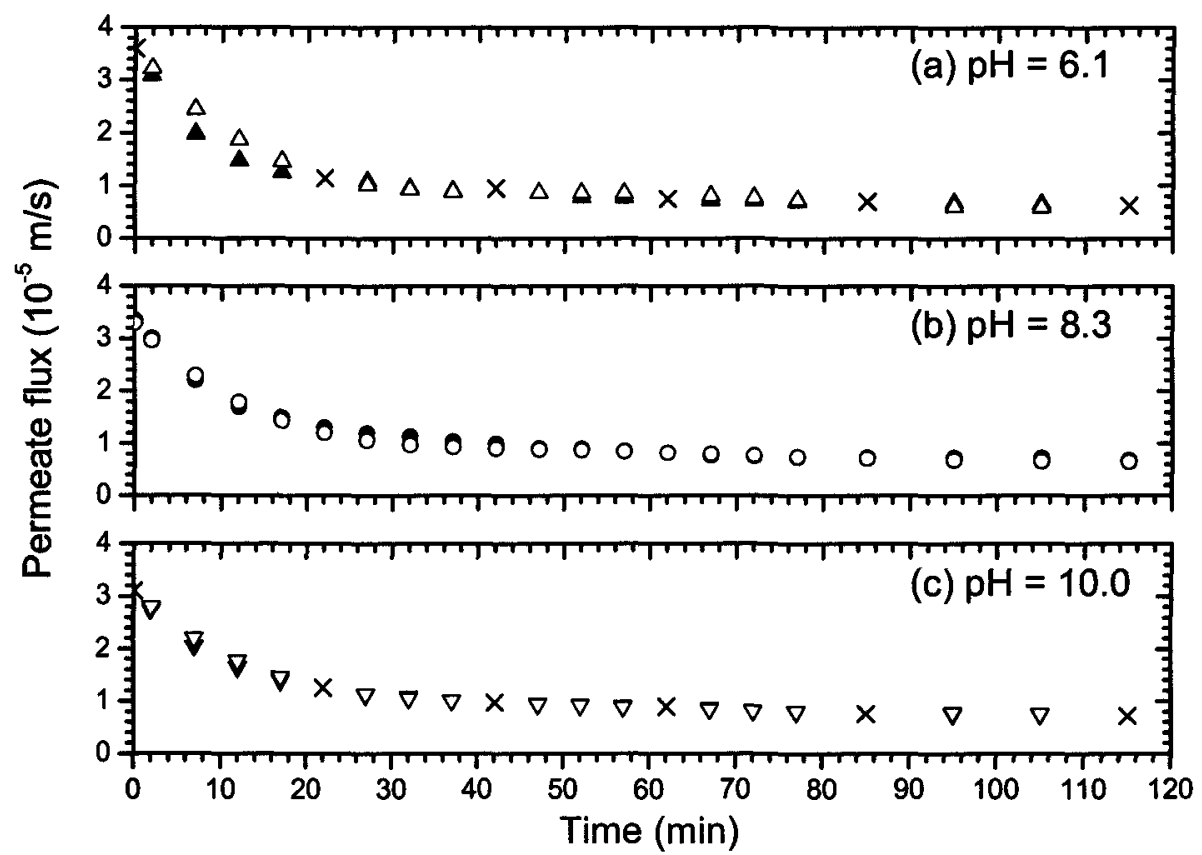

Figure 5. Experimental data and ANN predictions on effect of solution $\mathrm{pH}$ on permeate flux decline with ionic strength of $10^{-1} \mathrm{M} \mathrm{KCl}$. The filtration conditions employed are particle size $47 \mathrm{~nm}$, transmembrane pressure $41.4 \mathrm{kPa}$ (6 psi), particle volume concentration $0.01 \%$, cross flow velocity $0.246 \mathrm{~m} / \mathrm{s}$, and temperature $20{ }^{\circ} \mathrm{C}$. At each $\mathrm{pH}$ value, the solid and the hollow symbols represent experimentally observed and ANN simulated data while $x$ indicates data points used for the training process. 


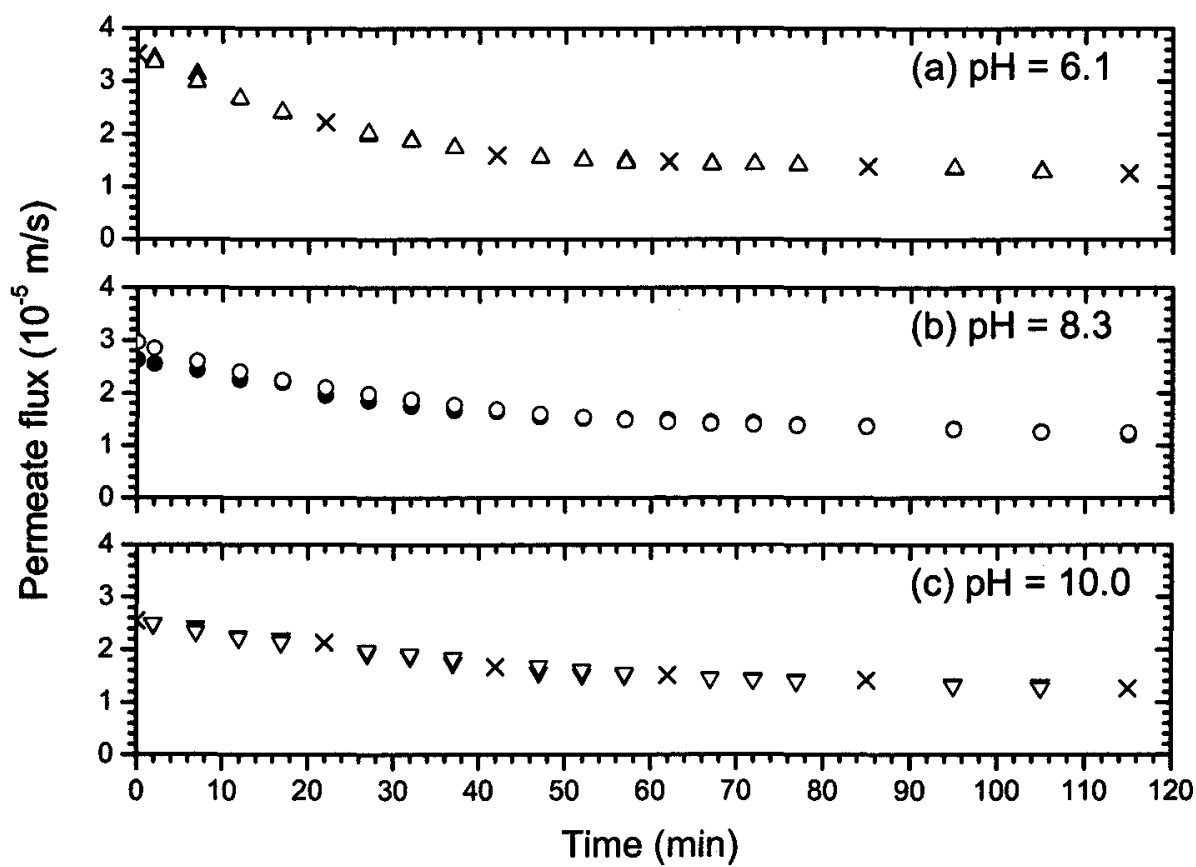

Figure 6. Experimental data and ANN predictions on the effect of solution $\mathrm{pH}$ on permeate flux decline with ionic strength of $10^{-3} \mathrm{M} \mathrm{KCl}$. The filtration conditions employed are particle size $47 \mathrm{~nm}$, transmembrane pressure $41.4 \mathrm{kPa}$ (6 psi), particle volume concentration $0.01 \%$, cross flow velocity $0.246 \mathrm{~m} / \mathrm{s}$, and temperature $20{ }^{\circ} \mathrm{C}$. At each $\mathrm{pH}$ value, the solid and the hollow symbols represent experimentally observed and ANN simulated data while $\times$ indicates data points used for the training process. 


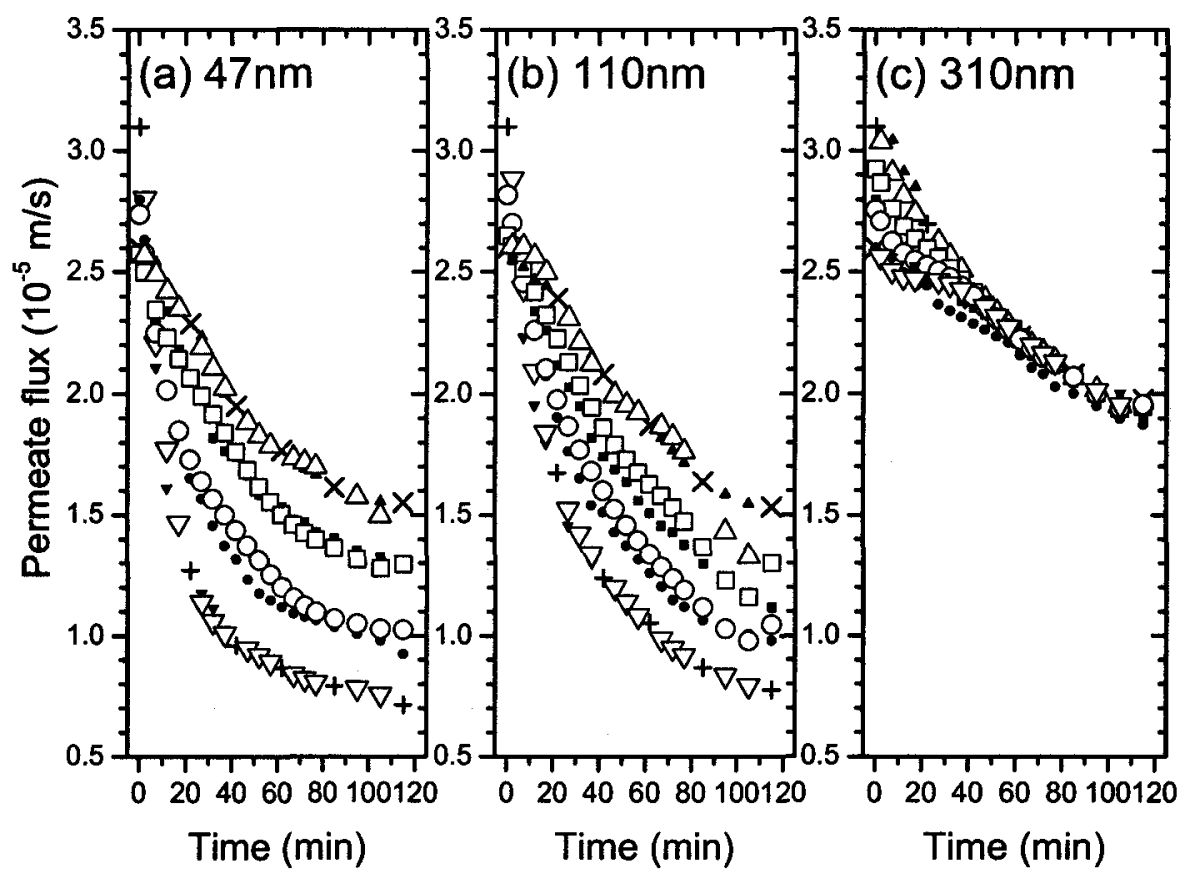

Figure 7. Experimental data and ANN predictions on the effect of ionic strength on permeate flux decline for (a) $47 \mathrm{~nm}$, (b) $110 \mathrm{~nm}$ and (c) $310 \mathrm{~nm}$ particles. The filtration conditions employed were $\mathrm{pH} 10$, transmembrane pressure $41.4 \mathrm{kPa}$ (6 psi), particle volume concentration $0.01 \%$, cross flow velocity $0.246 \mathrm{~m} / \mathrm{s}$, and temperature $20^{\circ} \mathrm{C}$. For each particle size, the symbol $\times$ and + indicate the selected data points of ionic strength of $10^{-4}$ and $10^{-1} M$, respectively, for the training process. The solid symbols indicate experimentally observed data of ionic strength $10^{-4} M(\Delta), 10^{-3} M(\square), 10^{-2} M(\bigcirc)$, and $10^{-1} M(\nabla)$ of $\mathrm{KCl}$ while the corresponding hollow symbols represent the simulation results. 


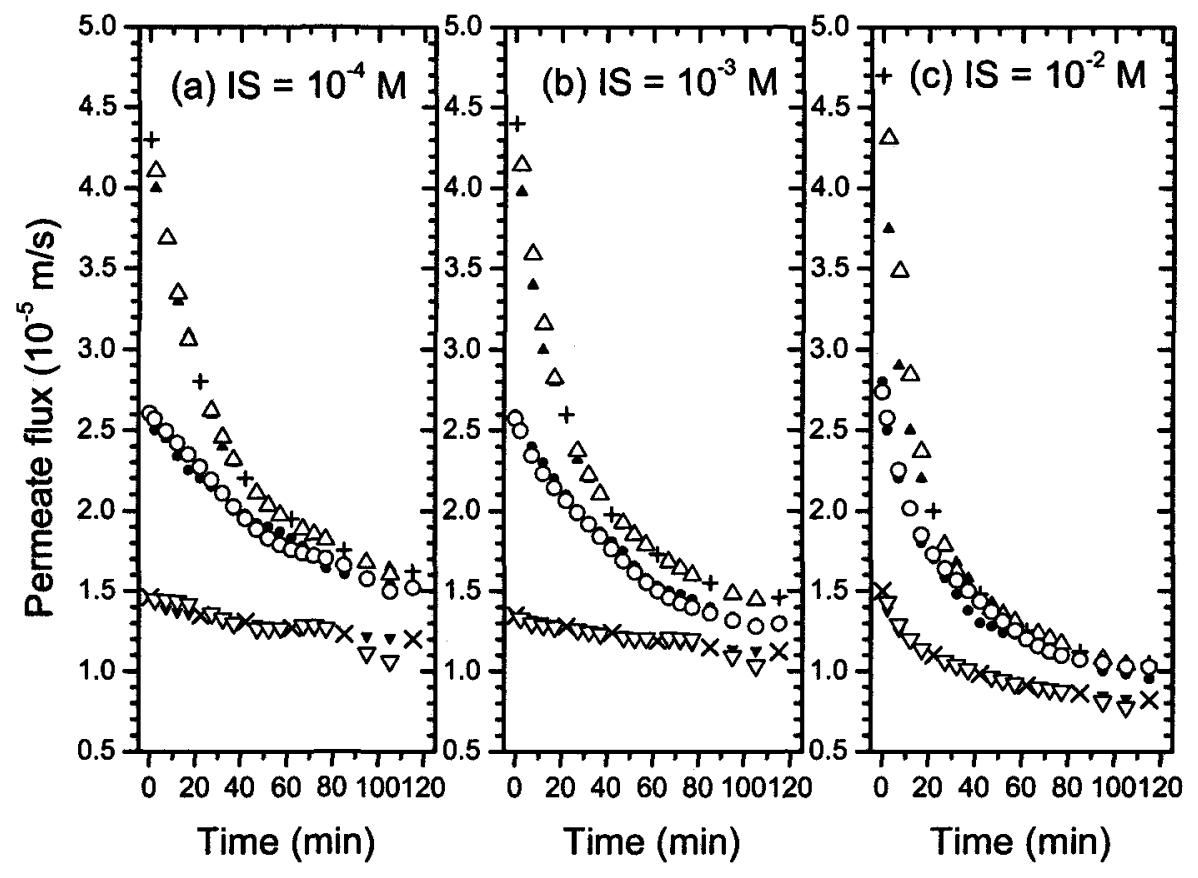

Figure 8. Experimental data and ANN predictions on the effect of transmembrane pressure on permeate flux decline for ionic strengths of (a) $10^{-4} \mathrm{M}$, (b) $10^{-3} \mathrm{M}$ and (c) $10^{-2}$ $M \mathrm{KCl}$. The filtration conditions employed were $\mathrm{pH} 10$, particle size $47 \mathrm{~nm}$, particle volume concentration $0.01 \%$, cross flow velocity $0.246 \mathrm{~m} / \mathrm{s}$, and temperature $20^{\circ} \mathrm{C}$. For each particle size, the symbol $x$ and + indicate the selected data points of transmembrane pressure of $62.1 \mathrm{kPa}(9 \mathrm{psi})$ and $20.7 \mathrm{kPa}(3 \mathrm{psi})$, respectively, for the training process. The solid symbols indicate experimentally observed data of transmembrane pressure 62.1 $\mathrm{kPa}(9 \mathrm{psi})(\mathbf{\Delta}), 41.4 \mathrm{kPa}(6 \mathrm{psi})(\mathbf{O})$, and $20.7 \mathrm{kPa}(3 \mathrm{psi})(\boldsymbol{\nabla})$ while the corresponding hollow symbols represent the simulation results. 


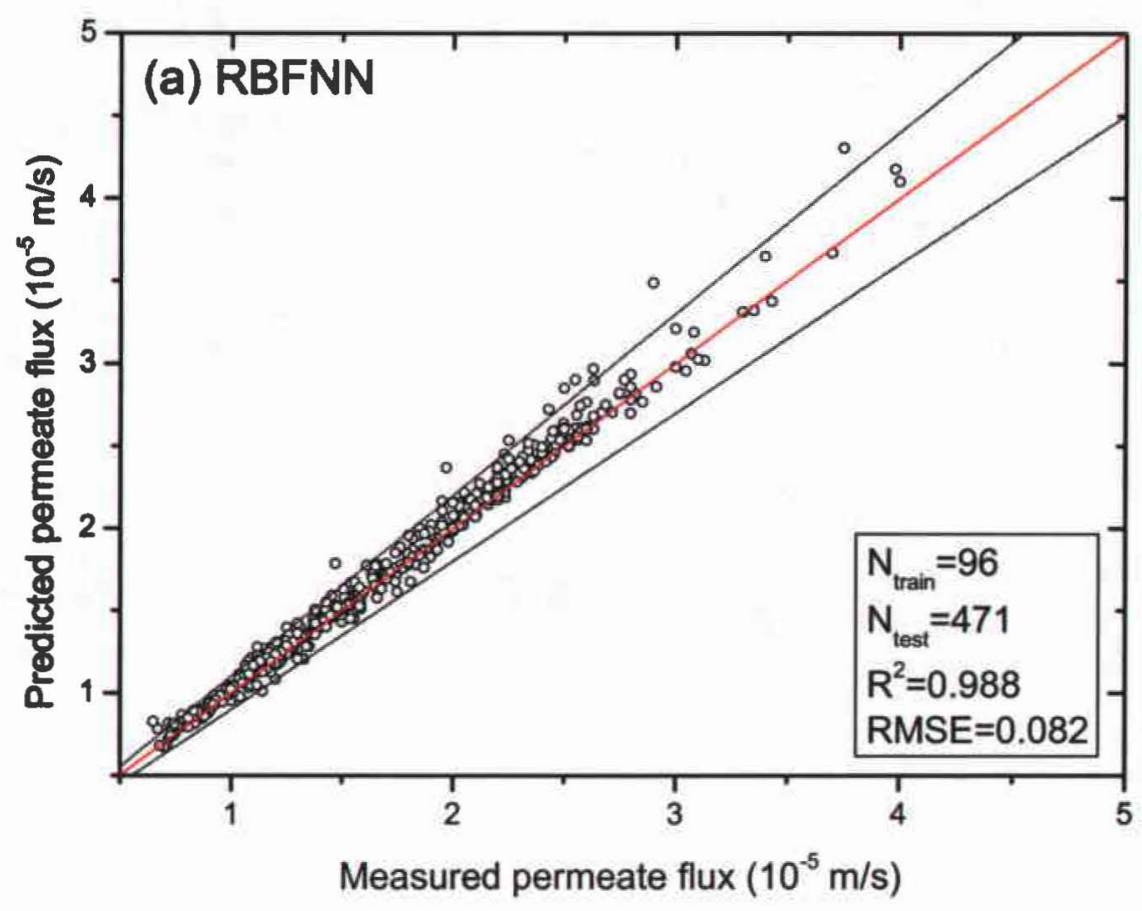

Figure 9(a). RBFNN predictions of permeate flux with experimental measurement under different operational conditions. The solid straight line represents the line of equality and the dashed lines represent $\pm 10 \%$ variance from it. The variance and RMSE between the predicted and measured data are also provided. 


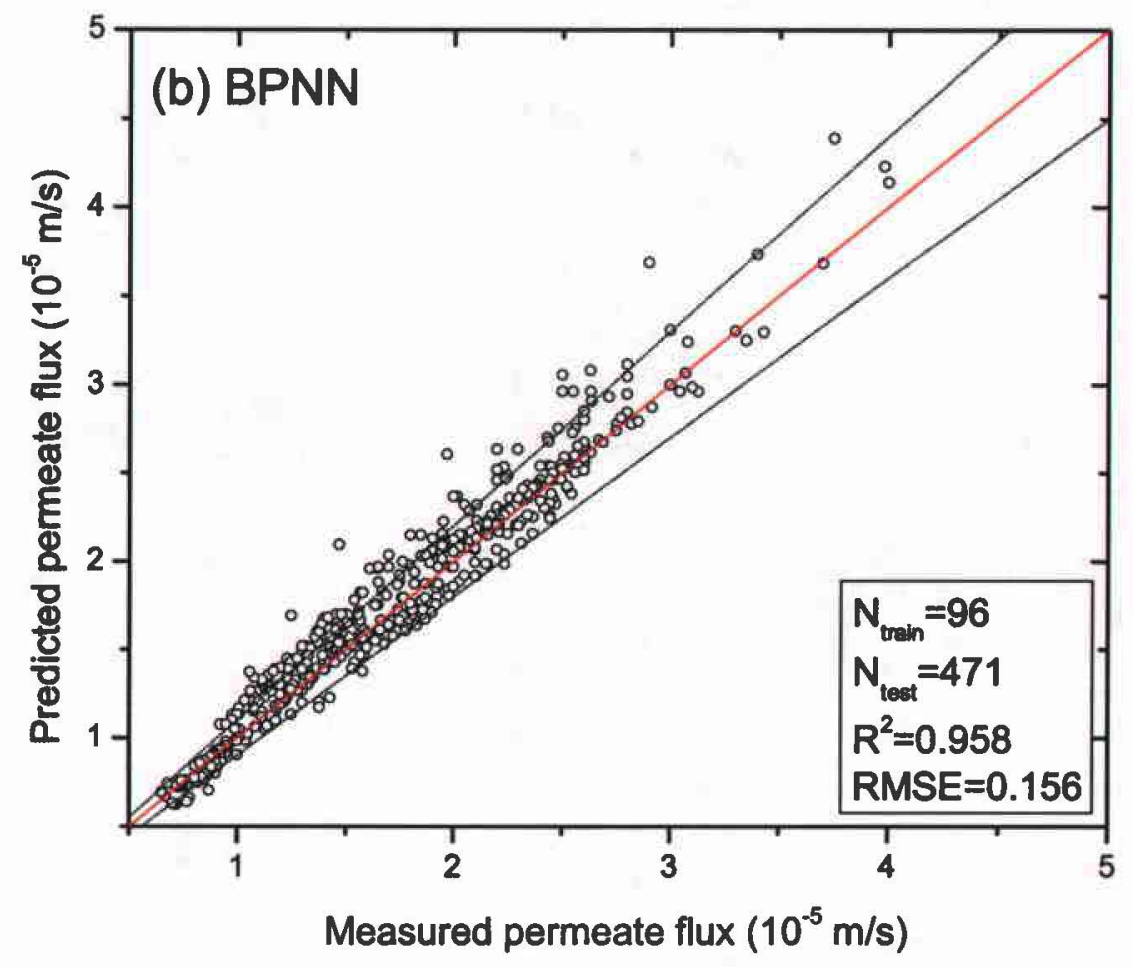

Figure 9(b). BPNN predictions of permeate flux with experimental measurement under different operational conditions. The solid straight line represents the line of equality and the dashed lines represent $\pm 10 \%$ variance from it. The variance and RMSE between the predicted and measured data are also provided. 


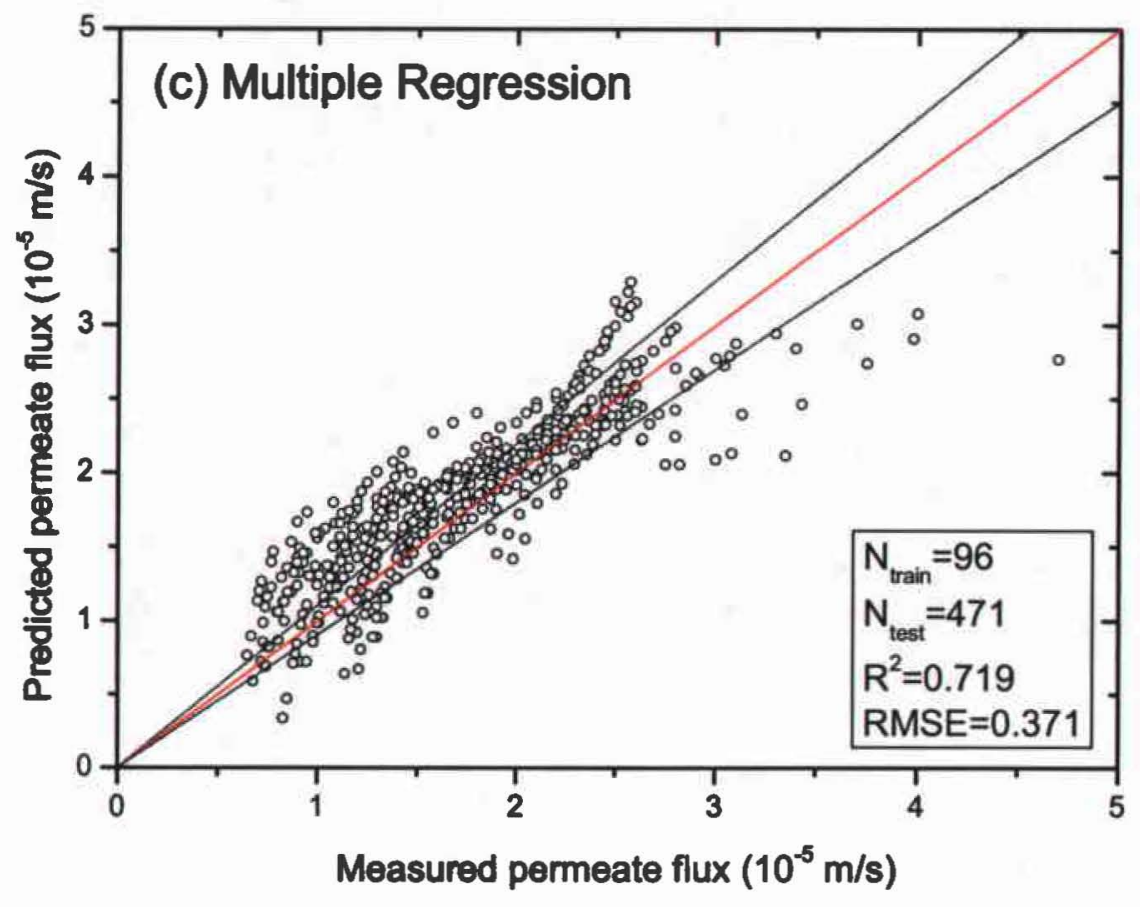

Figure $9(c)$. Multiple regression predictions of permeate flux with experimental measurement under different operational conditions. The solid straight line represents the line of equality and the dashed lines represent $\pm 10 \%$ variance from it. The variance and RMSE between the predicted and measured data are also provided. 


\section{APPENDIX A. A SUPPLEMENTARY CASE STUDY}

To examine whether a different ratio of data points in training and validation sets has any effect on the RBFNN's advantage, an additional case study was performed by using more training and less validation data points. Similar to the study presented in Chapter 4, a total of 567 data points from membrane experiments were used. Of these 567 data points, approximately $78 \%$ (441 data points) of all the experimental data were used for training and obtaining the interrelationship between inputs and outputs, and the remaining 22\% (126 data points) were used for model performance verification. As mentioned in Chapter 3, as all empirical models are only able to interpolate between the application boundary conditions (Shetty et al. 2003), the training data should be representative of the entire range of experimental conditions. Therefore, extreme values of the experimental data need to be included as part of the training set, in order to obtain better model prediction. In addition, it is always required to provide the same quality and quantity of data in order to compare the model performance. Thus, the results presented below all employed the same training and validation data sets.

Similarity, both RBFNN and BPNN model simulation applied in this supplementary case preserve the settings used in the study. The only difference is that the optimal "spread" number for RBFNN in the current case is 24 . The regression equation computed from the model has the following form:

$$
\hat{J}=0.712+0.024[\Delta P]+0.195[I S]-0.011[p H]+0.0027[P S]-0.012[t]
$$


Model performance was investigated by expressing the predicted data as a function of measured data (Figs. A1, A2, and A3). RBFNN, BPNN and multiple regression all exhibited better performance by providing more training data, when compared to the results in Chapter 4. This advantage of having more training data point was more evident in the BPNN, which showed an increased $R^{2}$ from 0.958 to 0.982 and decreased RMSE from 0.156 to 0.079 . In terms of the model performance, obviously, both RBFNN and BPNN produced better results than the multiple regression did, such that most of predicted data were within the $\pm 10 \%$ relative error. Multiple regression still cannot fully predict the nonlinear variation of permeate flux in membrane filtration, even given more sufficient training data points. By comparing the performance parameters of RBFNN and BPNN (Table. A1), one can still notice the advantage of RBFNN. Meanwhile, the superiority of RBFNN over BPNN is considerably reduced, showing relatively close values of $R^{2}$ and RMSE. 
Table A1. Comparison of the performance of RBFNN, BPNN and multiple regression for a supplementary case in terms of variance $\left(R^{2}\right)$, root mean square error (RMSE), number of points beyond $\pm 10 \%$ relative error $\left(N_{10}\right)$, and simulation time, given the same number of points for total data set $\left(N_{\text {total }}\right)$, training set $\left(N_{\text {train }}\right)$, and testing set $\left(N_{\text {test }}\right)$ for an additional case.

\begin{tabular}{c|c|c|c|c|c|c|c}
\hline & $N_{\text {total }}$ & $N_{\text {train }}$ & $N_{\text {test }}$ & $R^{2}$ & $\mathrm{RMSE}$ & $N_{10}$ & $\begin{array}{c}\text { Simulation } \\
\text { time (sec) }\end{array}$ \\
\hline RBFNN & 567 & 441 & 126 & 0.984 & 0.079 & 2 & 7.23 \\
\hline BPNN & 567 & 441 & 126 & 0.982 & 0.085 & 4 & 97 \\
\hline MR & 567 & 441 & 126 & 0.798 & 0.282 & 35 & 0.046 \\
\hline
\end{tabular}




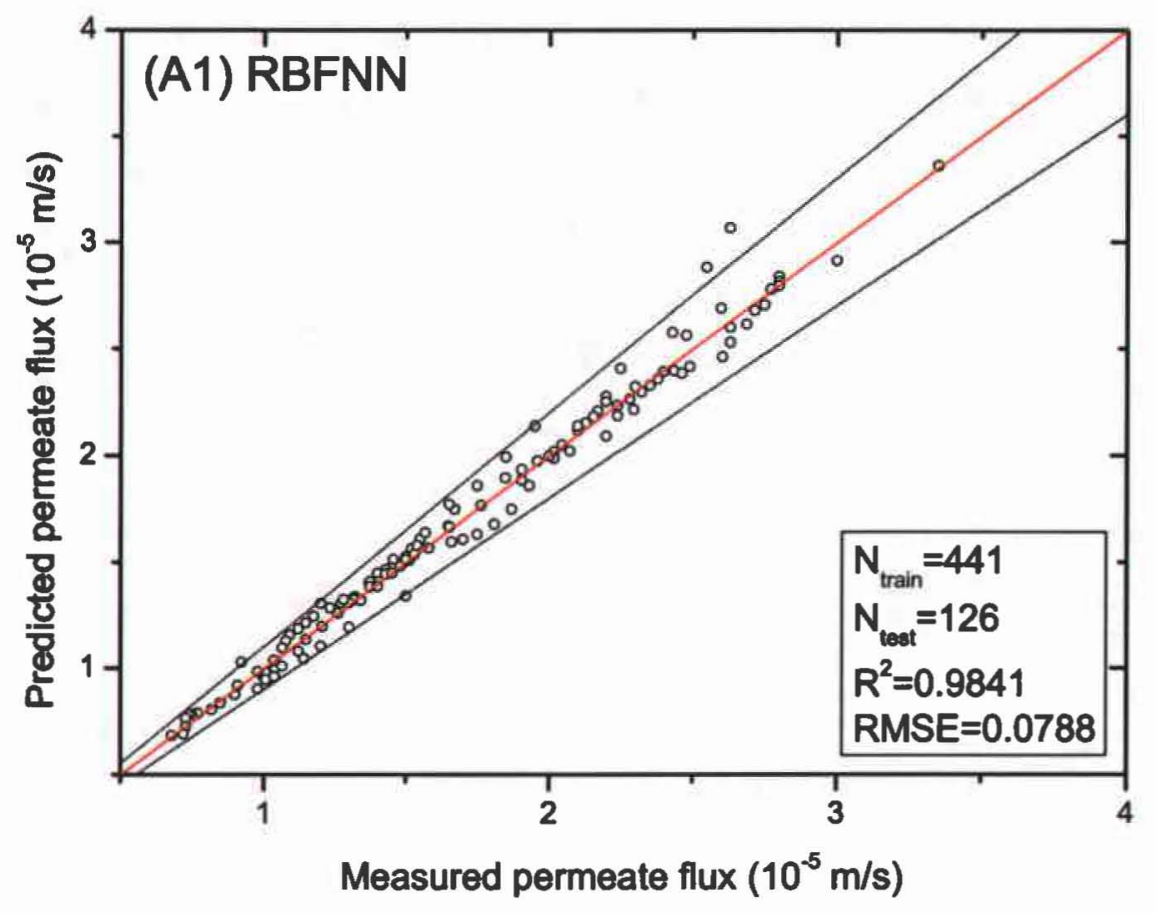

Figure A1 RBFNN predictions of permeate flux for a supplementary case. The solid straight line represents the line of equality and the dashed lines represent $\pm 10 \%$ variance from it. The variance and RMSE between the predicted and measured data are also provided. 


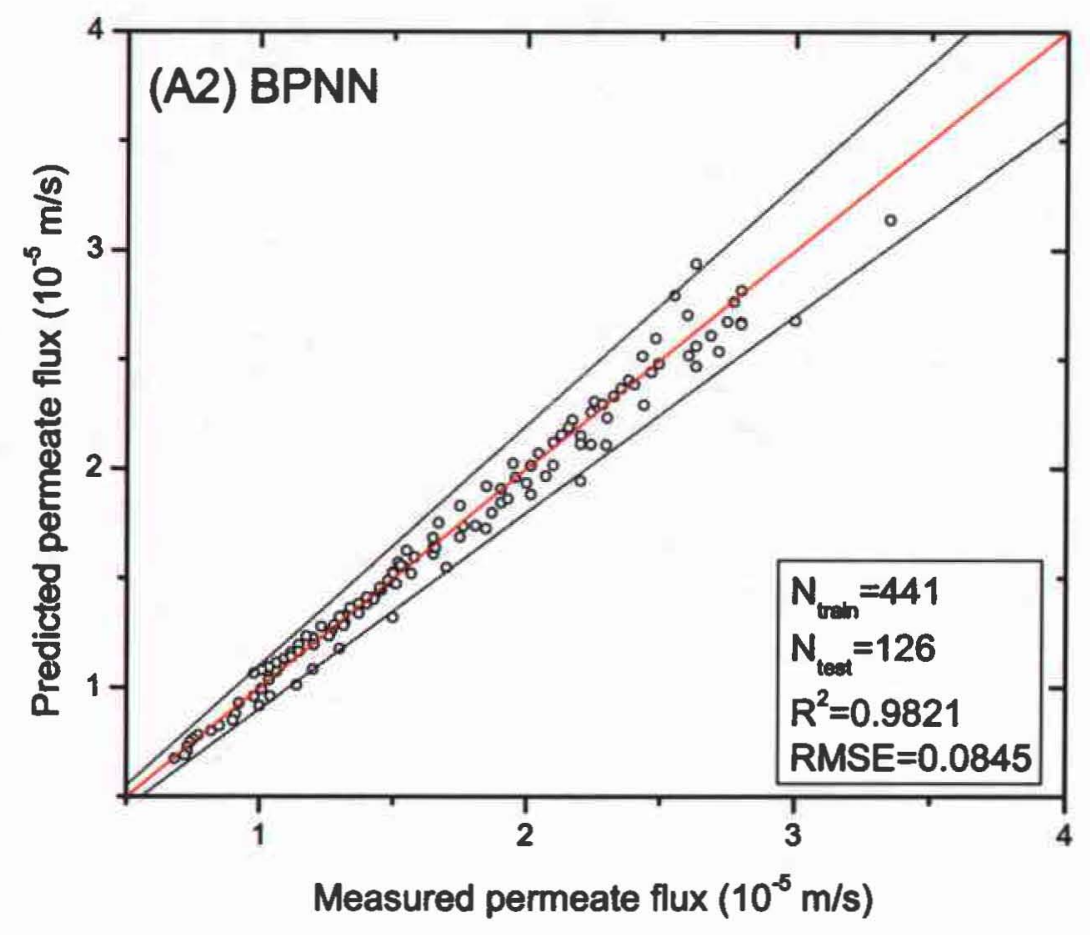

Figure A2 BPNN predictions of permeate flux for a supplementary case. The solid straight line represents the line of equality and the dashed lines represent $\pm 10 \%$ variance from it. The variance and RMSE between the predicted and measured data are also provided. 


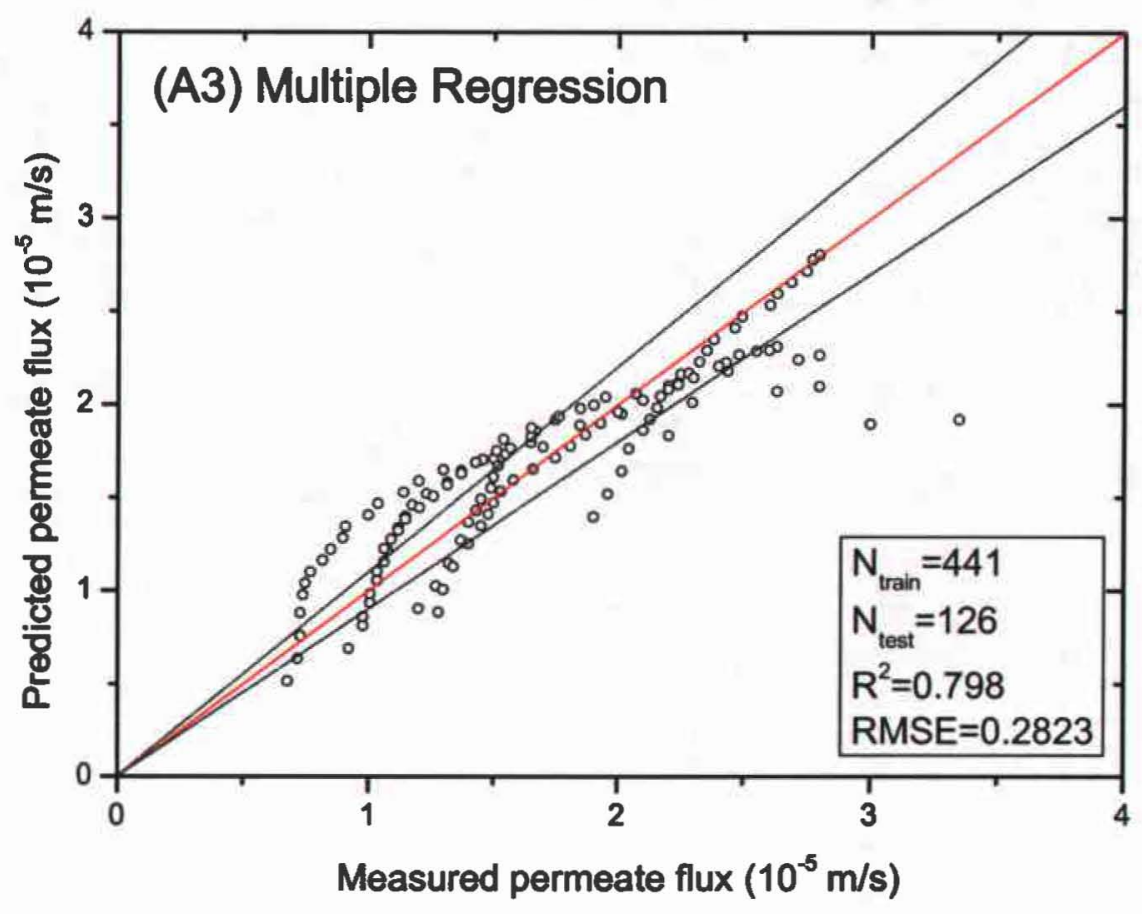

Figure A3 Multiple regression predictions of permeate flux for a supplementary case. The solid straight line represents the line of equality and the dashed lines represent $\pm 10 \%$ variance from it. The variance and RMSE between the predicted and measured data are also provided. 


\section{APPENDIX B. EXPERIMENTAL DATA (TRAINING SET)}

\begin{tabular}{|r|r|r|r|r|r|r|}
\hline \hline $\mathrm{NO}$ & $\mathrm{P}$ & $\mathrm{IS}$ & $\mathrm{pH}$ & $\mathrm{PS}$ & $\mathrm{t}$ & $\mathrm{J}$ \\
\hline & $\mathrm{kPs}$ & $\mathrm{M}$ & & $\mathrm{nm}$ & $\mathrm{min}$ & $\mathrm{m} / \mathrm{s}$ \\
\hline 1 & 41 & 1 & 6.1 & 47 & 0 & 3.6 \\
\hline 2 & 41 & 1 & 6.1 & 47 & 22 & 1.1 \\
\hline 3 & 41 & 1 & 6.1 & 47 & 42 & 1 \\
\hline 4 & 41 & 1 & 6.1 & 47 & 62 & 0.8 \\
\hline 5 & 41 & 1 & 6.1 & 47 & 85 & 0.7 \\
\hline 6 & 41 & 1 & 6.1 & 47 & 115 & 0.6 \\
\hline 7 & 41 & 1 & 10 & 47 & 0 & 3.1 \\
\hline 8 & 41 & 1 & 10 & 47 & 22 & 1.3 \\
\hline 9 & 41 & 1 & 10 & 47 & 42 & 1 \\
\hline 10 & 41 & 1 & 10 & 47 & 62 & 0.9 \\
\hline 11 & 41 & 1 & 10 & 47 & 85 & 0.8 \\
\hline 12 & 41 & 1 & 10 & 47 & 115 & 0.7 \\
\hline 13 & 41 & 3 & 6.1 & 47 & 0 & 3.5 \\
\hline 14 & 41 & 3 & 6.1 & 47 & 22 & 2.2 \\
\hline 15 & 41 & 3 & 6.1 & 47 & 42 & 1.6 \\
\hline 16 & 41 & 3 & 6.1 & 47 & 62 & 1.5 \\
\hline 17 & 41 & 3 & 6.1 & 47 & 85 & 1.4 \\
\hline 18 & 41 & 3 & 6.1 & 47 & 115 & 1.3 \\
\hline 19 & 41 & 3 & 10 & 47 & 0 & 2.6 \\
\hline 20 & 41 & 3 & 10 & 47 & 22 & 2.1 \\
\hline 21 & 41 & 3 & 10 & 47 & 42 & 1.7 \\
\hline 22 & 41 & 3 & 10 & 47 & 62 & 1.5 \\
\hline 23 & 41 & 3 & 10 & 47 & 85 & 1.4 \\
\hline 24 & 41 & 3 & 10 & 47 & 115 & 1.3 \\
\hline 25 & 41 & 1 & 10 & 47 & 0 & 3.1 \\
\hline 26 & 41 & 1 & 10 & 47 & 22 & 1.3 \\
\hline 27 & 41 & 1 & 10 & 47 & 42 & 1 \\
\hline 28 & 41 & 1 & 10 & 47 & 62 & 0.9 \\
\hline 29 & 41 & 1 & 10 & 47 & 85 & 0.8 \\
\hline 30 & 41 & 1 & 10 & 47 & 115 & 0.7 \\
\hline & & & & & \\
\hline
\end{tabular}

\begin{tabular}{|r|r|r|r|r|r|r|}
\hline \hline $\mathrm{NO}$ & $\mathrm{P}$ & $\mathrm{IS}$ & $\mathrm{pH}$ & $\mathrm{PS}$ & $\mathrm{t}$ & $\mathrm{J}$ \\
\hline & $\mathrm{kPs}$ & $\mathrm{M}$ & & $\mathrm{nm}$ & $\mathrm{min}$ & $\mathrm{m} / \mathrm{s}$ \\
\hline 31 & 41 & 4 & 10 & 47 & 0 & 2.6 \\
\hline 32 & 41 & 4 & 10 & 47 & 22 & 2.3 \\
\hline 33 & 41 & 4 & 10 & 47 & 42 & 2 \\
\hline 34 & 41 & 4 & 10 & 47 & 62 & 1.8 \\
\hline 35 & 41 & 4 & 10 & 47 & 85 & 1.6 \\
\hline 36 & 41 & 4 & 10 & 47 & 115 & 1.5 \\
\hline 37 & 41 & 1 & 10 & 110 & 0 & 3.1 \\
\hline 38 & 41 & 1 & 10 & 110 & 22 & 1.7 \\
\hline 39 & 41 & 1 & 10 & 110 & 42 & 1.2 \\
\hline 40 & 41 & 1 & 10 & 110 & 62 & 1.1 \\
\hline 41 & 41 & 1 & 10 & 110 & 85 & 0.9 \\
\hline 42 & 41 & 1 & 10 & 110 & 115 & 0.8 \\
\hline 43 & 41 & 4 & 10 & 110 & 0 & 2.6 \\
\hline 44 & 41 & 4 & 10 & 110 & 22 & 2.4 \\
\hline 45 & 41 & 4 & 10 & 110 & 42 & 2.1 \\
\hline 46 & 41 & 4 & 10 & 110 & 62 & 1.9 \\
\hline 47 & 41 & 4 & 10 & 110 & 85 & 1.6 \\
\hline 48 & 41 & 4 & 10 & 110 & 115 & 1.5 \\
\hline 49 & 41 & 1 & 10 & 310 & 0 & 3.1 \\
\hline 50 & 41 & 1 & 10 & 310 & 22 & 2.7 \\
\hline 51 & 41 & 1 & 10 & 310 & 42 & 2.4 \\
\hline 52 & 41 & 1 & 10 & 310 & 62 & 2.3 \\
\hline 53 & 41 & 1 & 10 & 310 & 85 & 2.1 \\
\hline 54 & 41 & 1 & 10 & 310 & 115 & 2 \\
\hline 55 & 41 & 4 & 10 & 310 & 0 & 2.6 \\
\hline 56 & 41 & 4 & 10 & 310 & 22 & 2.5 \\
\hline 57 & 41 & 4 & 10 & 310 & 42 & 2.4 \\
\hline 58 & 41 & 4 & 10 & 310 & 62 & 2.2 \\
\hline 59 & 41 & 4 & 10 & 310 & 85 & 2.1 \\
\hline 60 & 41 & 4 & 10 & 310 & 115 & 2 \\
\hline & & & & \\
\hline 5
\end{tabular}


Appendix B. (Continued) Experimental Data (Training Set)

\begin{tabular}{|r|r|r|r|r|r|r|}
\hline \hline $\mathrm{NO}$ & $\mathrm{P}$ & $\mathrm{IS}$ & $\mathrm{pH}$ & $\mathrm{PS}$ & $\mathrm{t}$ & $\mathrm{J}$ \\
\hline & $\mathrm{kPs}$ & $\mathrm{M}$ & & $\mathrm{nm}$ & $\mathrm{min}$ & $\mathrm{m} / \mathrm{s}$ \\
\hline 61 & 62 & 4 & 10 & 47 & 0 & 4.3 \\
\hline 62 & 62 & 4 & 10 & 47 & 22 & 2.8 \\
\hline 63 & 62 & 4 & 10 & 47 & 42 & 2.2 \\
\hline 64 & 62 & 4 & 10 & 47 & 62 & 2 \\
\hline 65 & 62 & 4 & 10 & 47 & 85 & 1.8 \\
\hline 66 & 62 & 4 & 10 & 47 & 115 & 1.6 \\
\hline 67 & 21 & 4 & 10 & 47 & 0 & 1.5 \\
\hline 68 & 21 & 4 & 10 & 47 & 22 & 1.4 \\
\hline 69 & 21 & 4 & 10 & 47 & 42 & 1.3 \\
\hline 70 & 21 & 4 & 10 & 47 & 62 & 1.3 \\
\hline 71 & 21 & 4 & 10 & 47 & 85 & 1.2 \\
\hline 72 & 21 & 4 & 10 & 47 & 115 & 1.2 \\
\hline 73 & 62 & 3 & 10 & 47 & 0 & 4.4 \\
\hline 74 & 62 & 3 & 10 & 47 & 22 & 2.6 \\
\hline 75 & 62 & 3 & 10 & 47 & 42 & 2 \\
\hline 76 & 62 & 3 & 10 & 47 & 62 & 1.7 \\
\hline 77 & 62 & 3 & 10 & 47 & 85 & 1.6 \\
\hline 78 & 62 & 3 & 10 & 47 & 115 & 1.5 \\
\hline 79 & 21 & 3 & 10 & 47 & 0 & 1.4 \\
\hline 80 & 21 & 3 & 10 & 47 & 22 & 1.3 \\
\hline 81 & 21 & 3 & 10 & 47 & 42 & 1.2 \\
\hline 82 & 21 & 3 & 10 & 47 & 62 & 1.2 \\
\hline 83 & 21 & 3 & 10 & 47 & 85 & 1.2 \\
\hline 84 & 21 & 3 & 10 & 47 & 115 & 1.1 \\
\hline 85 & 62 & 2 & 10 & 47 & 0 & 4.7 \\
\hline 86 & 62 & 2 & 10 & 47 & 22 & 2 \\
\hline 87 & 62 & 2 & 10 & 47 & 42 & 1.5 \\
\hline 88 & 62 & 2 & 10 & 47 & 62 & 1.3 \\
\hline 89 & 62 & 2 & 10 & 47 & 85 & 1.1 \\
\hline 90 & 62 & 2 & 10 & 47 & 115 & 1.1 \\
\hline
\end{tabular}

\begin{tabular}{|r|r|r|r|r|r|r|}
\hline \hline $\mathrm{NO}$ & $\mathrm{P}$ & $\mathrm{IS}$ & $\mathrm{pH}$ & $\mathrm{PS}$ & $\mathrm{t}$ & $\mathrm{J}$ \\
\hline & $\mathrm{kPs}$ & $\mathrm{M}$ & & $\mathrm{nm}$ & $\mathrm{min}$ & $\mathrm{m} / \mathrm{s}$ \\
\hline 91 & 21 & 2 & 10 & 47 & 0 & 1.5 \\
\hline 92 & 21 & 2 & 10 & 47 & 22 & 1.1 \\
\hline 93 & 21 & 2 & 10 & 47 & 42 & 1 \\
\hline 94 & 21 & 2 & 10 & 47 & 62 & 0.9 \\
\hline 95 & 21 & 2 & 10 & 47 & 85 & 0.9 \\
\hline 96 & 21 & 2 & 10 & 47 & 115 & 0.8 \\
\hline
\end{tabular}


APPENDIX C. EXPERIMENTAL DATA (VALIDATION SET)

\begin{tabular}{|r|r|r|r|r|r|r|}
\hline \hline $\mathrm{NO}$ & $\mathrm{P}$ & $\mathrm{IS}$ & $\mathrm{pH}$ & $\mathrm{PS}$ & $\mathrm{t}$ & $\mathrm{J}$ \\
\hline & $\mathrm{kPs}$ & $\mathrm{M}$ & & $\mathrm{nm}$ & $\mathrm{min}$ & $\mathrm{m} / \mathrm{s}$ \\
\hline 1 & 41.4 & 1 & 6.1 & 47 & 2 & 3.08 \\
\hline 2 & 41.4 & 1 & 6.1 & 47 & 7 & 1.97 \\
\hline 3 & 41.4 & 1 & 6.1 & 47 & 12 & 1.47 \\
\hline 4 & 41.4 & 1 & 6.1 & 47 & 17 & 1.25 \\
\hline 5 & 41.4 & 1 & 6.1 & 47 & 27 & 1.08 \\
\hline 6 & 41.4 & 1 & 6.1 & 47 & 32 & 0.95 \\
\hline 7 & 41.4 & 1 & 6.1 & 47 & 37 & 0.9 \\
\hline 8 & 41.4 & 1 & 6.1 & 47 & 47 & 0.87 \\
\hline 9 & 41.4 & 1 & 6.1 & 47 & 52 & 0.78 \\
\hline 10 & 41.4 & 1 & 6.1 & 47 & 57 & 0.77 \\
\hline 11 & 41.4 & 1 & 6.1 & 47 & 67 & 0.72 \\
\hline 12 & 41.4 & 1 & 6.1 & 47 & 72 & 0.71 \\
\hline 13 & 41.4 & 1 & 6.1 & 47 & 77 & 0.7 \\
\hline 14 & 41.4 & 1 & 6.1 & 47 & 95 & 0.67 \\
\hline 15 & 41.4 & 1 & 6.1 & 47 & 105 & 0.65 \\
\hline 16 & 41.4 & 1 & 8.3 & 47 & 0 & 3.35 \\
\hline 17 & 41.4 & 1 & 8.3 & 47 & 2 & 3 \\
\hline 18 & 41.4 & 1 & 8.3 & 47 & 7 & 2.2 \\
\hline 19 & 41.4 & 1 & 8.3 & 47 & 12 & 1.7 \\
\hline 20 & 41.4 & 1 & 8.3 & 47 & 17 & 1.5 \\
\hline 21 & 41.4 & 1 & 8.3 & 47 & 22 & 1.3 \\
\hline 22 & 41.4 & 1 & 8.3 & 47 & 27 & 1.2 \\
\hline 23 & 41.4 & 1 & 8.3 & 47 & 32 & 1.14 \\
\hline 24 & 41.4 & 1 & 8.3 & 47 & 37 & 1.04 \\
\hline 25 & 41.4 & 1 & 8.3 & 47 & 42 & 1 \\
\hline 26 & 41.4 & 1 & 8.3 & 47 & 47 & 0.91 \\
\hline 27 & 41.4 & 1 & 8.3 & 47 & 52 & 0.9 \\
\hline 28 & 41.4 & 1 & 8.3 & 47 & 57 & 0.85 \\
\hline 29 & 41.4 & 1 & 8.3 & 47 & 62 & 0.82 \\
\hline 30 & 41.4 & 1 & 8.3 & 47 & 67 & 0.77 \\
\hline 31 & 41.4 & 1 & 8.3 & 47 & 72 & 0.75 \\
\hline 32 & 41.4 & 1 & 8.3 & 47 & 77 & 0.74 \\
\hline 33 & 41.4 & 1 & 8.3 & 47 & 85 & 0.73 \\
\hline 34 & 41.4 & 1 & 8.3 & 47 & 95 & 0.73 \\
\hline 35 & 41.4 & 1 & 8.3 & 47 & 105 & 0.72 \\
\hline
\end{tabular}

\begin{tabular}{|r|r|r|r|r|r|r|}
\hline NO & $\mathrm{P}$ & $\mathrm{IS}$ & $\mathrm{pH}$ & $\mathrm{PS}$ & $\mathrm{t}$ & $\mathrm{J}$ \\
\hline & $\mathrm{kPs}$ & $\mathrm{M}$ & & $\mathrm{nm}$ & $\mathrm{min}$ & $\mathrm{m} / \mathrm{s}$ \\
\hline 36 & 41.4 & 1 & 8.3 & 47 & 115 & 0.68 \\
\hline 37 & 41.4 & 1 & 10 & 47 & 2 & 2.75 \\
\hline 38 & 41.4 & 1 & 10 & 47 & 7 & 2.05 \\
\hline 39 & 41.4 & 1 & 10 & 47 & 12 & 1.65 \\
\hline 40 & 41.4 & 1 & 10 & 47 & 17 & 1.4 \\
\hline 41 & 41.4 & 1 & 10 & 47 & 27 & 1.125 \\
\hline 42 & 41.4 & 1 & 10 & 47 & 32 & 1.09 \\
\hline 43 & 41.4 & 1 & 10 & 47 & 37 & 1.01 \\
\hline 44 & 41.4 & 1 & 10 & 47 & 47 & 0.95 \\
\hline 45 & 41.4 & 1 & 10 & 47 & 52 & 0.93 \\
\hline 46 & 41.4 & 1 & 10 & 47 & 57 & 0.91 \\
\hline 47 & 41.4 & 1 & 10 & 47 & 67 & 0.87 \\
\hline 48 & 41.4 & 1 & 10 & 47 & 72 & 0.83 \\
\hline 49 & 41.4 & 1 & 10 & 47 & 77 & 0.8 \\
\hline 50 & 41.4 & 1 & 10 & 47 & 95 & 0.75 \\
\hline 51 & 41.4 & 1 & 10 & 47 & 105 & 0.74 \\
\hline 52 & 41.4 & 3 & 6.1 & 47 & 2 & 3.43 \\
\hline 53 & 41.4 & 3 & 6.1 & 47 & 7 & 3.13 \\
\hline 54 & 41.4 & 3 & 6.1 & 47 & 12 & 2.67 \\
\hline 55 & 41.4 & 3 & 6.1 & 47 & 17 & 2.4 \\
\hline 56 & 41.4 & 3 & 6.1 & 47 & 27 & 1.98 \\
\hline 57 & 41.4 & 3 & 6.1 & 47 & 32 & 1.89 \\
\hline 58 & 41.4 & 3 & 6.1 & 47 & 37 & 1.75 \\
\hline 59 & 41.4 & 3 & 6.1 & 47 & 47 & 1.56 \\
\hline 60 & 41.4 & 3 & 6.1 & 47 & 52 & 1.51 \\
\hline 61 & 41.4 & 3 & 6.1 & 47 & 57 & 1.5 \\
\hline 62 & 41.4 & 3 & 6.1 & 47 & 67 & 1.43 \\
\hline 63 & 41.4 & 3 & 6.1 & 47 & 72 & 1.42 \\
\hline 64 & 41.4 & 3 & 6.1 & 47 & 77 & 1.41 \\
\hline 65 & 41.4 & 3 & 6.1 & 47 & 95 & 1.34 \\
\hline 66 & 41.4 & 3 & 6.1 & 47 & 105 & 1.3 \\
\hline 67 & 41.4 & 3 & 8.3 & 47 & 0 & 2.63 \\
\hline 68 & 41.4 & 3 & 8.3 & 47 & 2 & 2.55 \\
\hline 69 & 41.4 & 3 & 8.3 & 47 & 7 & 2.43 \\
\hline 70 & 41.4 & 3 & 8.3 & 47 & 12 & 2.25 \\
\hline
\end{tabular}


Appendix C. (Continued) Experimental Data (Prediction Set)

\begin{tabular}{|r|r|r|r|r|r|r|}
\hline \hline $\mathrm{NO}$ & $\mathrm{P}$ & $\mathrm{IS}$ & $\mathrm{pH}$ & $\mathrm{PS}$ & $\mathrm{t}$ & $\mathrm{J}$ \\
\hline & $\mathrm{kPs}$ & $\mathrm{M}$ & & $\mathrm{nm}$ & $\mathrm{min}$ & $\mathrm{m} / \mathrm{s}$ \\
\hline 71 & 41.4 & 3 & 8.3 & 47 & 17 & 2.2 \\
\hline 72 & 41.4 & 3 & 8.3 & 47 & 22 & 1.95 \\
\hline 73 & 41.4 & 3 & 8.3 & 47 & 27 & 1.85 \\
\hline 74 & 41.4 & 3 & 8.3 & 47 & 32 & 1.75 \\
\hline 75 & 41.4 & 3 & 8.3 & 47 & 37 & 1.67 \\
\hline 76 & 41.4 & 3 & 8.3 & 47 & 42 & 1.65 \\
\hline 77 & 41.4 & 3 & 8.3 & 47 & 47 & 1.55 \\
\hline 78 & 41.4 & 3 & 8.3 & 47 & 52 & 1.52 \\
\hline 79 & 41.4 & 3 & 8.3 & 47 & 57 & 1.5 \\
\hline 80 & 41.4 & 3 & 8.3 & 47 & 62 & 1.49 \\
\hline 81 & 41.4 & 3 & 8.3 & 47 & 67 & 1.45 \\
\hline 82 & 41.4 & 3 & 8.3 & 47 & 72 & 1.43 \\
\hline 83 & 41.4 & 3 & 8.3 & 47 & 77 & 1.4 \\
\hline 84 & 41.4 & 3 & 8.3 & 47 & 85 & 1.37 \\
\hline 85 & 41.4 & 3 & 8.3 & 47 & 95 & 1.32 \\
\hline 86 & 41.4 & 3 & 8.3 & 47 & 105 & 1.27 \\
\hline 87 & 41.4 & 3 & 8.3 & 47 & 115 & 1.2 \\
\hline 88 & 41.4 & 3 & 10 & 47 & 2 & 2.48 \\
\hline 89 & 41.4 & 3 & 10 & 47 & 7 & 2.44 \\
\hline 90 & 41.4 & 3 & 10 & 47 & 12 & 2.26 \\
\hline 91 & 41.4 & 3 & 10 & 47 & 17 & 2.21 \\
\hline 92 & 41.4 & 3 & 10 & 47 & 27 & 1.93 \\
\hline 93 & 41.4 & 3 & 10 & 47 & 32 & 1.87 \\
\hline 94 & 41.4 & 3 & 10 & 47 & 37 & 1.76 \\
\hline 95 & 41.4 & 3 & 10 & 47 & 47 & 1.57 \\
\hline 96 & 41.4 & 3 & 10 & 47 & 52 & 1.53 \\
\hline 97 & 41.4 & 3 & 10 & 47 & 57 & 1.52 \\
\hline 98 & 41.4 & 3 & 10 & 47 & 67 & 1.46 \\
\hline 99 & 41.4 & 3 & 10 & 47 & 72 & 1.45 \\
\hline 100 & 41.4 & 3 & 10 & 47 & 77 & 1.43 \\
\hline 101 & 41.4 & 3 & 10 & 47 & 95 & 1.35 \\
\hline 102 & 41.4 & 3 & 10 & 47 & 105 & 1.33 \\
\hline 103 & 41.4 & 1 & 10 & 47 & 2 & 2.821 \\
\hline 104 & 41.4 & 1 & 10 & 47 & 7 & 2.108 \\
\hline 105 & 41.4 & 1 & 10 & 47 & 12 & 1.612 \\
\hline
\end{tabular}

\begin{tabular}{|r|r|r|r|r|r|r|}
\hline \hline NO & P & IS & pH & PS & $\mathrm{t}$ & $\mathrm{J}$ \\
\hline & $\mathrm{kPs}$ & $\mathrm{M}$ & & $\mathrm{nm}$ & $\mathrm{min}$ & $\mathrm{m} / \mathrm{s}$ \\
\hline 106 & 41.4 & 1 & 10 & 47 & 17 & 1.457 \\
\hline 107 & 41.4 & 1 & 10 & 47 & 27 & 1.178 \\
\hline 108 & 41.4 & 1 & 10 & 47 & 32 & 1.116 \\
\hline 109 & 41.4 & 1 & 10 & 47 & 37 & 0.992 \\
\hline 110 & 41.4 & 1 & 10 & 47 & 47 & 0.93 \\
\hline 111 & 41.4 & 1 & 10 & 47 & 52 & 0.899 \\
\hline 112 & 41.4 & 1 & 10 & 47 & 57 & 0.884 \\
\hline 113 & 41.4 & 1 & 10 & 47 & 67 & 0.853 \\
\hline 114 & 41.4 & 1 & 10 & 47 & 72 & 0.837 \\
\hline 115 & 41.4 & 1 & 10 & 47 & 77 & 0.806 \\
\hline 116 & 41.4 & 1 & 10 & 47 & 95 & 0.76 \\
\hline 117 & 41.4 & 1 & 10 & 47 & 105 & 0.744 \\
\hline 118 & 41.4 & 2 & 10 & 47 & 0 & 2.8 \\
\hline 119 & 41.4 & 2 & 10 & 47 & 2 & 2.632 \\
\hline 120 & 41.4 & 2 & 10 & 47 & 7 & 2.296 \\
\hline 121 & 41.4 & 2 & 10 & 47 & 12 & 2.016 \\
\hline 122 & 41.4 & 2 & 10 & 47 & 17 & 1.848 \\
\hline 123 & 41.4 & 2 & 10 & 47 & 22 & 1.652 \\
\hline 124 & 41.4 & 2 & 10 & 47 & 27 & 1.568 \\
\hline 125 & 41.4 & 2 & 10 & 47 & 32 & 1.456 \\
\hline 126 & 41.4 & 2 & 10 & 47 & 37 & 1.372 \\
\hline 127 & 41.4 & 2 & 10 & 47 & 42 & 1.316 \\
\hline 128 & 41.4 & 2 & 10 & 47 & 47 & 1.232 \\
\hline 129 & 41.4 & 2 & 10 & 47 & 52 & 1.176 \\
\hline 130 & 41.4 & 2 & 10 & 47 & 57 & 1.148 \\
\hline 131 & 41.4 & 2 & 10 & 47 & 62 & 1.12 \\
\hline 132 & 41.4 & 2 & 10 & 47 & 67 & 1.092 \\
\hline 133 & 41.4 & 2 & 10 & 47 & 72 & 1.078 \\
\hline 134 & 41.4 & 2 & 10 & 47 & 77 & 1.064 \\
\hline 135 & 41.4 & 2 & 10 & 47 & 85 & 1.036 \\
\hline 136 & 41.4 & 2 & 10 & 47 & 95 & 1.008 \\
\hline 137 & 41.4 & 2 & 10 & 47 & 105 & 0.98 \\
\hline 138 & 41.4 & 2 & 10 & 47 & 115 & 0.924 \\
\hline 139 & 41.4 & 3 & 10 & 47 & 0 & 2.6 \\
\hline 140 & 41.4 & 3 & 10 & 47 & 2 & 2.561 \\
\hline
\end{tabular}


Appendix C. (Continued) Experimental Data (Prediction Set)

\begin{tabular}{|r|r|r|r|r|r|r|}
\hline NO & $\mathrm{P}$ & $\mathrm{IS}$ & $\mathrm{pH}$ & $\mathrm{PS}$ & $\mathrm{t}$ & $\mathrm{J}$ \\
\hline & $\mathrm{kPs}$ & $\mathrm{M}$ & & $\mathrm{nm}$ & $\mathrm{min}$ & $\mathrm{m} / \mathrm{s}$ \\
\hline 141 & 41.4 & 3 & 10 & 47 & 7 & 2.522 \\
\hline 142 & 41.4 & 3 & 10 & 47 & 12 & 2.34 \\
\hline 143 & 41.4 & 3 & 10 & 47 & 17 & 2.184 \\
\hline 144 & 41.4 & 3 & 10 & 47 & 22 & 2.054 \\
\hline 145 & 41.4 & 3 & 10 & 47 & 27 & 1.989 \\
\hline 146 & 41.4 & 3 & 10 & 47 & 32 & 1.82 \\
\hline 147 & 41.4 & 3 & 10 & 47 & 37 & 1.768 \\
\hline 148 & 41.4 & 3 & 10 & 47 & 42 & 1.742 \\
\hline 149 & 41.4 & 3 & 10 & 47 & 47 & 1.664 \\
\hline 150 & 41.4 & 3 & 10 & 47 & 52 & 1.586 \\
\hline 151 & 41.4 & 3 & 10 & 47 & 57 & 1.573 \\
\hline 152 & 41.4 & 3 & 10 & 47 & 62 & 1.534 \\
\hline 153 & 41.4 & 3 & 10 & 47 & 67 & 1.482 \\
\hline 154 & 41.4 & 3 & 10 & 47 & 72 & 1.469 \\
\hline 155 & 41.4 & 3 & 10 & 47 & 77 & 1.43 \\
\hline 156 & 41.4 & 3 & 10 & 47 & 85 & 1.404 \\
\hline 157 & 41.4 & 3 & 10 & 47 & 95 & 1.352 \\
\hline 158 & 41.4 & 3 & 10 & 47 & 105 & 1.326 \\
\hline 159 & 41.4 & 3 & 10 & 47 & 115 & 1.3 \\
\hline 160 & 41.4 & 4 & 10 & 47 & 2 & 2.574 \\
\hline 161 & 41.4 & 4 & 10 & 47 & 7 & 2.543 \\
\hline 162 & 41.4 & 4 & 10 & 47 & 12 & 2.444 \\
\hline 163 & 41.4 & 4 & 10 & 47 & 17 & 2.366 \\
\hline 164 & 41.4 & 4 & 10 & 47 & 27 & 2.236 \\
\hline 165 & 41.4 & 4 & 10 & 47 & 32 & 2.106 \\
\hline 166 & 41.4 & 4 & 10 & 47 & 37 & 2.002 \\
\hline 167 & 41.4 & 4 & 10 & 47 & 47 & 1.872 \\
\hline 168 & 41.4 & 4 & 10 & 47 & 52 & 1.846 \\
\hline 169 & 41.4 & 4 & 10 & 47 & 57 & 1.794 \\
\hline 170 & 41.4 & 4 & 10 & 47 & 67 & 1.742 \\
\hline 171 & 41.4 & 4 & 10 & 47 & 72 & 1.69 \\
\hline 172 & 41.4 & 4 & 10 & 47 & 77 & 1.664 \\
\hline 173 & 41.4 & 4 & 10 & 47 & 95 & 1.586 \\
\hline 174 & 41.4 & 4 & 10 & 47 & 105 & 1.555 \\
\hline 175 & 41.4 & 1 & 10 & 110 & 2 & 2.635 \\
\hline
\end{tabular}

\begin{tabular}{|r|r|r|r|r|r|r|}
\hline \hline $\mathrm{NO}$ & $\mathrm{P}$ & $\mathrm{IS}$ & $\mathrm{pH}$ & $\mathrm{PS}$ & $\mathrm{t}$ & $\mathrm{J}$ \\
\hline & $\mathrm{kPs}$ & $\mathrm{M}$ & & $\mathrm{nm}$ & $\mathrm{min}$ & $\mathrm{m} / \mathrm{s}$ \\
\hline 176 & 41.4 & 1 & 10 & 110 & 7 & 2.232 \\
\hline 177 & 41.4 & 1 & 10 & 110 & 12 & 1.953 \\
\hline 178 & 41.4 & 1 & 10 & 110 & 17 & 1.798 \\
\hline 179 & 41.4 & 1 & 10 & 110 & 27 & 1.457 \\
\hline 180 & 41.4 & 1 & 10 & 110 & 32 & 1.395 \\
\hline 181 & 41.4 & 1 & 10 & 110 & 37 & 1.302 \\
\hline 182 & 41.4 & 1 & 10 & 110 & 47 & 1.178 \\
\hline 183 & 41.4 & 1 & 10 & 110 & 52 & 1.116 \\
\hline 184 & 41.4 & 1 & 10 & 110 & 57 & 1.085 \\
\hline 185 & 41.4 & 1 & 10 & 110 & 67 & 0.992 \\
\hline 186 & 41.4 & 1 & 10 & 110 & 72 & 0.961 \\
\hline 187 & 41.4 & 1 & 10 & 110 & 77 & 0.899 \\
\hline 188 & 41.4 & 1 & 10 & 110 & 95 & 0.837 \\
\hline 189 & 41.4 & 1 & 10 & 110 & 105 & 0.806 \\
\hline 190 & 41.4 & 2 & 10 & 110 & 0 & 2.8 \\
\hline 191 & 41.4 & 2 & 10 & 110 & 2 & 2.716 \\
\hline 192 & 41.4 & 2 & 10 & 110 & 7 & 2.436 \\
\hline 193 & 41.4 & 2 & 10 & 110 & 12 & 2.24 \\
\hline 194 & 41.4 & 2 & 10 & 110 & 17 & 2.072 \\
\hline 195 & 41.4 & 2 & 10 & 110 & 22 & 1.904 \\
\hline 196 & 41.4 & 2 & 10 & 110 & 27 & 1.764 \\
\hline 197 & 41.4 & 2 & 10 & 110 & 32 & 1.652 \\
\hline 198 & 41.4 & 2 & 10 & 110 & 37 & 1.54 \\
\hline 199 & 41.4 & 2 & 10 & 110 & 42 & 1.512 \\
\hline 200 & 41.4 & 2 & 10 & 110 & 47 & 1.428 \\
\hline 201 & 41.4 & 2 & 10 & 110 & 52 & 1.372 \\
\hline 202 & 41.4 & 2 & 10 & 110 & 57 & 1.316 \\
\hline 203 & 41.4 & 2 & 10 & 110 & 62 & 1.26 \\
\hline 204 & 41.4 & 2 & 10 & 110 & 67 & 1.204 \\
\hline 205 & 41.4 & 2 & 10 & 110 & 72 & 1.148 \\
\hline 206 & 41.4 & 2 & 10 & 110 & 77 & 1.12 \\
\hline 207 & 41.4 & 2 & 10 & 110 & 85 & 1.064 \\
\hline 208 & 41.4 & 2 & 10 & 110 & 95 & 1.036 \\
\hline 209 & 41.4 & 2 & 10 & 110 & 105 & 1.008 \\
\hline 210 & 41.4 & 2 & 10 & 110 & 115 & 0.98 \\
\hline
\end{tabular}


Appendix C. (Continued) Experimental Data (Prediction Set)

\begin{tabular}{|r|r|r|r|r|r|r|}
\hline \hline NO & $\mathrm{P}$ & $\mathrm{IS}$ & $\mathrm{pH}$ & $\mathrm{PS}$ & $\mathrm{t}$ & $\mathrm{J}$ \\
\hline & $\mathrm{kPS}$ & $\mathrm{M}$ & & $\mathrm{nm}$ & $\mathrm{min}$ & $\mathrm{m} / \mathrm{s}$ \\
\hline 211 & 41.4 & 3 & 10 & 110 & 0 & 2.6 \\
\hline 212 & 41.4 & 3 & 10 & 110 & 2 & 2.548 \\
\hline 213 & 41.4 & 3 & 10 & 110 & 7 & 2.517 \\
\hline 214 & 41.4 & 3 & 10 & 110 & 12 & 2.34 \\
\hline 215 & 41.4 & 3 & 10 & 110 & 17 & 2.262 \\
\hline 216 & 41.4 & 3 & 10 & 110 & 22 & 2.119 \\
\hline 217 & 41.4 & 3 & 10 & 110 & 27 & 2.028 \\
\hline 218 & 41.4 & 3 & 10 & 110 & 32 & 1.95 \\
\hline 219 & 41.4 & 3 & 10 & 110 & 37 & 1.82 \\
\hline 220 & 41.4 & 3 & 10 & 110 & 42 & 1.742 \\
\hline 221 & 41.4 & 3 & 10 & 110 & 47 & 1.69 \\
\hline 222 & 41.4 & 3 & 10 & 110 & 52 & 1.638 \\
\hline 223 & 41.4 & 3 & 10 & 110 & 57 & 1.56 \\
\hline 224 & 41.4 & 3 & 10 & 110 & 62 & 1.508 \\
\hline 225 & 41.4 & 3 & 10 & 110 & 67 & 1.456 \\
\hline 226 & 41.4 & 3 & 10 & 110 & 72 & 1.43 \\
\hline 227 & 41.4 & 3 & 10 & 110 & 77 & 1.378 \\
\hline 228 & 41.4 & 3 & 10 & 110 & 85 & 1.3 \\
\hline 229 & 41.4 & 3 & 10 & 110 & 95 & 1.248 \\
\hline 230 & 41.4 & 3 & 10 & 110 & 105 & 1.17 \\
\hline 231 & 41.4 & 3 & 10 & 110 & 115 & 1.118 \\
\hline 232 & 41.4 & 4 & 10 & 110 & 2 & 2.548 \\
\hline 233 & 41.4 & 4 & 10 & 110 & 7 & 2.522 \\
\hline 234 & 41.4 & 4 & 10 & 110 & 12 & 2.47 \\
\hline 235 & 41.4 & 4 & 10 & 110 & 17 & 2.444 \\
\hline 236 & 41.4 & 4 & 10 & 110 & 27 & 2.314 \\
\hline 237 & 41.4 & 4 & 10 & 110 & 32 & 2.236 \\
\hline 238 & 41.4 & 4 & 10 & 110 & 37 & 2.158 \\
\hline 239 & 41.4 & 4 & 10 & 110 & 47 & 2.028 \\
\hline 240 & 41.4 & 4 & 10 & 110 & 52 & 1.976 \\
\hline 241 & 41.4 & 4 & 10 & 110 & 57 & 1.924 \\
\hline 242 & 41.4 & 4 & 10 & 110 & 67 & 1.82 \\
\hline 243 & 41.4 & 4 & 10 & 110 & 72 & 1.768 \\
\hline 244 & 41.4 & 4 & 10 & 110 & 77 & 1.716 \\
\hline 245 & 41.4 & 4 & 10 & 110 & 95 & 1.586 \\
\hline
\end{tabular}

\begin{tabular}{|r|r|r|r|r|r|r|}
\hline NO & P & IS & pH & PS & t & J \\
\hline & $\mathrm{kPs}$ & $\mathrm{M}$ & & $\mathrm{nm}$ & $\mathrm{min}$ & $\mathrm{m} / \mathrm{s}$ \\
\hline 246 & 41.4 & 4 & 10 & 110 & 105 & 1.547 \\
\hline 247 & 41.4 & 1 & 10 & 310 & 2 & 3.069 \\
\hline 248 & 41.4 & 1 & 10 & 310 & 7 & 3.044 \\
\hline 249 & 41.4 & 1 & 10 & 310 & 12 & 2.914 \\
\hline 250 & 41.4 & 1 & 10 & 310 & 17 & 2.852 \\
\hline 251 & 41.4 & 1 & 10 & 310 & 27 & 2.604 \\
\hline 252 & 41.4 & 1 & 10 & 310 & 32 & 2.511 \\
\hline 253 & 41.4 & 1 & 10 & 310 & 37 & 2.449 \\
\hline 254 & 41.4 & 1 & 10 & 310 & 47 & 2.387 \\
\hline 255 & 41.4 & 1 & 10 & 310 & 52 & 2.356 \\
\hline 256 & 41.4 & 1 & 10 & 310 & 57 & 2.294 \\
\hline 257 & 41.4 & 1 & 10 & 310 & 67 & 2.232 \\
\hline 258 & 41.4 & 1 & 10 & 310 & 72 & 2.201 \\
\hline 259 & 41.4 & 1 & 10 & 310 & 77 & 2.108 \\
\hline 260 & 41.4 & 1 & 10 & 310 & 95 & 2.046 \\
\hline 261 & 41.4 & 1 & 10 & 310 & 105 & 1.984 \\
\hline 262 & 41.4 & 2 & 10 & 310 & 0 & 2.8 \\
\hline 263 & 41.4 & 2 & 10 & 310 & 2 & 2.772 \\
\hline 264 & 41.4 & 2 & 10 & 310 & 7 & 2.75 \\
\hline 265 & 41.4 & 2 & 10 & 310 & 12 & 2.688 \\
\hline 266 & 41.4 & 2 & 10 & 310 & 17 & 2.632 \\
\hline 267 & 41.4 & 2 & 10 & 310 & 22 & 2.604 \\
\hline 268 & 41.4 & 2 & 10 & 310 & 27 & 2.492 \\
\hline 269 & 41.4 & 2 & 10 & 310 & 32 & 2.464 \\
\hline 270 & 41.4 & 2 & 10 & 310 & 37 & 2.38 \\
\hline 271 & 41.4 & 2 & 10 & 310 & 42 & 2.352 \\
\hline 272 & 41.4 & 2 & 10 & 310 & 47 & 2.324 \\
\hline 273 & 41.4 & 2 & 10 & 310 & 52 & 2.282 \\
\hline 274 & 41.4 & 2 & 10 & 310 & 57 & 2.24 \\
\hline 275 & 41.4 & 2 & 10 & 310 & 62 & 2.17 \\
\hline 276 & 41.4 & 2 & 10 & 310 & 67 & 2.156 \\
\hline 277 & 41.4 & 2 & 10 & 310 & 72 & 2.128 \\
\hline 278 & 41.4 & 2 & 10 & 310 & 77 & 2.1 \\
\hline 279 & 41.4 & 2 & 10 & 310 & 85 & 2.044 \\
\hline 280 & 41.4 & 2 & 10 & 310 & 95 & 2.016 \\
\hline
\end{tabular}


Appendix C. (Continued) Experimental Data (Prediction Set)

\begin{tabular}{|r|r|r|r|r|r|r|}
\hline \hline $\mathrm{NO}$ & $\mathrm{P}$ & $\mathrm{IS}$ & $\mathrm{pH}$ & $\mathrm{PS}$ & $\mathrm{t}$ & $\mathrm{J}$ \\
\hline & $\mathrm{kPs}$ & $\mathrm{M}$ & & $\mathrm{nm}$ & $\mathrm{min}$ & $\mathrm{m} / \mathrm{s}$ \\
\hline 281 & 41.4 & 2 & 10 & 310 & 105 & 1.96 \\
\hline 282 & 41.4 & 2 & 10 & 310 & 115 & 1.904 \\
\hline 283 & 41.4 & 3 & 10 & 310 & 0 & 2.6 \\
\hline 284 & 41.4 & 3 & 10 & 310 & 2 & 2.574 \\
\hline 285 & 41.4 & 3 & 10 & 310 & 7 & 2.558 \\
\hline 286 & 41.4 & 3 & 10 & 310 & 12 & 2.496 \\
\hline 287 & 41.4 & 3 & 10 & 310 & 17 & 2.457 \\
\hline 288 & 41.4 & 3 & 10 & 310 & 22 & 2.444 \\
\hline 289 & 41.4 & 3 & 10 & 310 & 27 & 2.366 \\
\hline 290 & 41.4 & 3 & 10 & 310 & 32 & 2.34 \\
\hline 291 & 41.4 & 3 & 10 & 310 & 37 & 2.314 \\
\hline 292 & 41.4 & 3 & 10 & 310 & 42 & 2.288 \\
\hline 293 & 41.4 & 3 & 10 & 310 & 47 & 2.262 \\
\hline 294 & 41.4 & 3 & 10 & 310 & 52 & 2.236 \\
\hline 295 & 41.4 & 3 & 10 & 310 & 57 & 2.21 \\
\hline 296 & 41.4 & 3 & 10 & 310 & 62 & 2.158 \\
\hline 297 & 41.4 & 3 & 10 & 310 & 67 & 2.106 \\
\hline 298 & 41.4 & 3 & 10 & 310 & 72 & 2.08 \\
\hline 299 & 41.4 & 3 & 10 & 310 & 77 & 2.028 \\
\hline 300 & 41.4 & 3 & 10 & 310 & 85 & 2.002 \\
\hline 301 & 41.4 & 3 & 10 & 310 & 95 & 1.95 \\
\hline 302 & 41.4 & 3 & 10 & 310 & 105 & 1.898 \\
\hline 303 & 41.4 & 3 & 10 & 310 & 115 & 1.872 \\
\hline 304 & 41.4 & 4 & 10 & 310 & 2 & 2.574 \\
\hline 305 & 41.4 & 4 & 10 & 310 & 7 & 2.561 \\
\hline 306 & 41.4 & 4 & 10 & 310 & 12 & 2.496 \\
\hline 307 & 41.4 & 4 & 10 & 310 & 17 & 2.522 \\
\hline 308 & 41.4 & 4 & 10 & 310 & 27 & 2.457 \\
\hline 309 & 41.4 & 4 & 10 & 310 & 32 & 2.444 \\
\hline 310 & 41.4 & 4 & 10 & 310 & 37 & 2.418 \\
\hline 311 & 41.4 & 4 & 10 & 310 & 47 & 2.366 \\
\hline 312 & 41.4 & 4 & 10 & 310 & 52 & 2.314 \\
\hline 313 & 41.4 & 4 & 10 & 310 & 57 & 2.288 \\
\hline 314 & 41.4 & 4 & 10 & 310 & 67 & 2.21 \\
\hline 315 & 41.4 & 4 & 10 & 310 & 72 & 2.158 \\
\hline
\end{tabular}

\begin{tabular}{|r|r|r|r|r|r|r|}
\hline \hline $\mathrm{NO}$ & $\mathrm{P}$ & $\mathrm{IS}$ & $\mathrm{pH}$ & $\mathrm{PS}$ & $\mathrm{t}$ & $\mathrm{J}$ \\
\hline & $\mathrm{kPs}$ & $\mathrm{M}$ & & $\mathrm{nm}$ & $\mathrm{min}$ & $\mathrm{m} / \mathrm{s}$ \\
\hline 316 & 41.4 & 4 & 10 & 310 & 77 & 2.106 \\
\hline 317 & 41.4 & 4 & 10 & 310 & 95 & 2.028 \\
\hline 318 & 41.4 & 4 & 10 & 310 & 105 & 2.002 \\
\hline 319 & 62.1 & 4 & 10 & 47 & 2 & 4 \\
\hline 320 & 62.1 & 4 & 10 & 47 & 7 & 3.7 \\
\hline 321 & 62.1 & 4 & 10 & 47 & 12 & 3.3 \\
\hline 322 & 62.1 & 4 & 10 & 47 & 17 & 3.1 \\
\hline 323 & 62.1 & 4 & 10 & 47 & 27 & 2.6 \\
\hline 324 & 62.1 & 4 & 10 & 47 & 32 & 2.4 \\
\hline 325 & 62.1 & 4 & 10 & 47 & 37 & 2.3 \\
\hline 326 & 62.1 & 4 & 10 & 47 & 47 & 2.1 \\
\hline 327 & 62.1 & 4 & 10 & 47 & 52 & 2.05 \\
\hline 328 & 62.1 & 4 & 10 & 47 & 57 & 2 \\
\hline 329 & 62.1 & 4 & 10 & 47 & 67 & 1.9 \\
\hline 330 & 62.1 & 4 & 10 & 47 & 72 & 1.86 \\
\hline 331 & 62.1 & 4 & 10 & 47 & 77 & 1.81 \\
\hline 332 & 62.1 & 4 & 10 & 47 & 95 & 1.7 \\
\hline 333 & 62.1 & 4 & 10 & 47 & 105 & 1.65 \\
\hline 334 & 41.4 & 4 & 10 & 47 & 0 & 2.6 \\
\hline 335 & 41.4 & 4 & 10 & 47 & 2 & 2.5 \\
\hline 336 & 41.4 & 4 & 10 & 47 & 7 & 2.45 \\
\hline 337 & 41.4 & 4 & 10 & 47 & 12 & 2.34 \\
\hline 338 & 41.4 & 4 & 10 & 47 & 17 & 2.25 \\
\hline 339 & 41.4 & 4 & 10 & 47 & 22 & 2.2 \\
\hline 340 & 41.4 & 4 & 10 & 47 & 27 & 2.15 \\
\hline 341 & 41.4 & 4 & 10 & 47 & 32 & 2.1 \\
\hline 342 & 41.4 & 4 & 10 & 47 & 37 & 2 \\
\hline 343 & 41.4 & 4 & 10 & 47 & 42 & 1.98 \\
\hline 344 & 41.4 & 4 & 10 & 47 & 47 & 1.92 \\
\hline 345 & 41.4 & 4 & 10 & 47 & 52 & 1.9 \\
\hline 346 & 41.4 & 4 & 10 & 47 & 57 & 1.87 \\
\hline 347 & 41.4 & 4 & 10 & 47 & 62 & 1.83 \\
\hline 348 & 41.4 & 4 & 10 & 47 & 67 & 1.78 \\
\hline 349 & 41.4 & 4 & 10 & 47 & 72 & 1.72 \\
\hline 350 & 41.4 & 4 & 10 & 47 & 77 & 1.64 \\
\hline
\end{tabular}


Appendix C. (Continued) Experimental Data (Prediction Set)

\begin{tabular}{|r|r|r|r|r|r|r|}
\hline \hline $\mathrm{NO}$ & $\mathrm{P}$ & $\mathrm{IS}$ & $\mathrm{pH}$ & $\mathrm{PS}$ & $\mathrm{t}$ & $\mathrm{J}$ \\
\hline & $\mathrm{kPs}$ & $\mathrm{M}$ & & $\mathrm{nm}$ & $\mathrm{min}$ & $\mathrm{m} / \mathrm{s}$ \\
\hline 351 & 41.4 & 4 & 10 & 47 & 85 & 1.6 \\
\hline 352 & 41.4 & 4 & 10 & 47 & 95 & 1.57 \\
\hline 353 & 41.4 & 4 & 10 & 47 & 105 & 1.54 \\
\hline 354 & 41.4 & 4 & 10 & 47 & 115 & 1.53 \\
\hline 355 & 20.7 & 4 & 10 & 47 & 2 & 1.42 \\
\hline 356 & 20.7 & 4 & 10 & 47 & 7 & 1.39 \\
\hline 357 & 20.7 & 4 & 10 & 47 & 12 & 1.37 \\
\hline 358 & 20.7 & 4 & 10 & 47 & 17 & 1.36 \\
\hline 359 & 20.7 & 4 & 10 & 47 & 27 & 1.34 \\
\hline 360 & 20.7 & 4 & 10 & 47 & 32 & 1.33 \\
\hline 361 & 20.7 & 4 & 10 & 47 & 37 & 1.32 \\
\hline 362 & 20.7 & 4 & 10 & 47 & 47 & 1.3 \\
\hline 363 & 20.7 & 4 & 10 & 47 & 52 & 1.29 \\
\hline 364 & 20.7 & 4 & 10 & 47 & 57 & 1.28 \\
\hline 365 & 20.7 & 4 & 10 & 47 & 67 & 1.26 \\
\hline 366 & 20.7 & 4 & 10 & 47 & 72 & 1.25 \\
\hline 367 & 20.7 & 4 & 10 & 47 & 77 & 1.24 \\
\hline 368 & 20.7 & 4 & 10 & 47 & 95 & 1.22 \\
\hline 369 & 20.7 & 4 & 10 & 47 & 105 & 1.21 \\
\hline 370 & 62.1 & 3 & 10 & 47 & 2 & 3.98 \\
\hline 371 & 62.1 & 3 & 10 & 47 & 7 & 3.4 \\
\hline 372 & 62.1 & 3 & 10 & 47 & 12 & 3 \\
\hline 373 & 62.1 & 3 & 10 & 47 & 17 & 2.8 \\
\hline 374 & 62.1 & 3 & 10 & 47 & 27 & 2.32 \\
\hline 375 & 62.1 & 3 & 10 & 47 & 32 & 2.2 \\
\hline 376 & 62.1 & 3 & 10 & 47 & 37 & 2.1 \\
\hline 377 & 62.1 & 3 & 10 & 47 & 47 & 1.92 \\
\hline 378 & 62.1 & 3 & 10 & 47 & 52 & 1.85 \\
\hline 379 & 62.1 & 3 & 10 & 47 & 57 & 1.8 \\
\hline 380 & 62.1 & 3 & 10 & 47 & 67 & 1.7 \\
\hline 381 & 62.1 & 3 & 10 & 47 & 72 & 1.65 \\
\hline 382 & 62.1 & 3 & 10 & 47 & 77 & 1.6 \\
\hline 383 & 62.1 & 3 & 10 & 47 & 95 & 1.48 \\
\hline 384 & 62.1 & 3 & 10 & 47 & 105 & 1.47 \\
\hline 385 & 41.4 & 3 & 10 & 47 & 0 & 2.6 \\
\hline
\end{tabular}

\begin{tabular}{|r|r|r|r|r|r|r|}
\hline $\mathrm{NO}$ & $\mathrm{P}$ & $\mathrm{IS}$ & $\mathrm{pH}$ & $\mathrm{PS}$ & $\mathrm{t}$ & $\mathrm{J}$ \\
\hline & $\mathrm{kPs}$ & $\mathrm{M}$ & & $\mathrm{nm}$ & $\mathrm{min}$ & $\mathrm{m} / \mathrm{s}$ \\
\hline 386 & 41.4 & 3 & 10 & 47 & 2 & 2.48 \\
\hline 387 & 41.4 & 3 & 10 & 47 & 7 & 2.4 \\
\hline 388 & 41.4 & 3 & 10 & 47 & 12 & 2.3 \\
\hline 389 & 41.4 & 3 & 10 & 47 & 17 & 2.2 \\
\hline 390 & 41.4 & 3 & 10 & 47 & 22 & 2.1 \\
\hline 391 & 41.4 & 3 & 10 & 47 & 27 & 2 \\
\hline 392 & 41.4 & 3 & 10 & 47 & 32 & 1.93 \\
\hline 393 & 41.4 & 3 & 10 & 47 & 37 & 1.87 \\
\hline 394 & 41.4 & 3 & 10 & 47 & 42 & 1.81 \\
\hline 395 & 41.4 & 3 & 10 & 47 & 47 & 1.75 \\
\hline 396 & 41.4 & 3 & 10 & 47 & 52 & 1.66 \\
\hline 397 & 41.4 & 3 & 10 & 47 & 57 & 1.58 \\
\hline 398 & 41.4 & 3 & 10 & 47 & 62 & 1.53 \\
\hline 399 & 41.4 & 3 & 10 & 47 & 67 & 1.5 \\
\hline 400 & 41.4 & 3 & 10 & 47 & 72 & 1.48 \\
\hline 401 & 41.4 & 3 & 10 & 47 & 77 & 1.45 \\
\hline 402 & 41.4 & 3 & 10 & 47 & 85 & 1.4 \\
\hline 403 & 41.4 & 3 & 10 & 47 & 95 & 1.34 \\
\hline 404 & 41.4 & 3 & 10 & 47 & 105 & 1.3 \\
\hline 405 & 41.4 & 3 & 10 & 47 & 115 & 1.28 \\
\hline 406 & 20.7 & 3 & 10 & 47 & 2 & 1.34 \\
\hline 407 & 20.7 & 3 & 10 & 47 & 7 & 1.33 \\
\hline 408 & 20.7 & 3 & 10 & 47 & 12 & 1.32 \\
\hline 409 & 20.7 & 3 & 10 & 47 & 17 & 1.3 \\
\hline 410 & 20.7 & 3 & 10 & 47 & 27 & 1.27 \\
\hline 411 & 20.7 & 3 & 10 & 47 & 32 & 1.26 \\
\hline 412 & 20.7 & 3 & 10 & 47 & 37 & 1.25 \\
\hline 413 & 20.7 & 3 & 10 & 47 & 47 & 1.23 \\
\hline 414 & 20.7 & 3 & 10 & 47 & 52 & 1.22 \\
\hline 415 & 20.7 & 3 & 10 & 47 & 57 & 1.2 \\
\hline 416 & 20.7 & 3 & 10 & 47 & 67 & 1.18 \\
\hline 417 & 20.7 & 3 & 10 & 47 & 72 & 1.17 \\
\hline 418 & 20.7 & 3 & 10 & 47 & 77 & 1.16 \\
\hline 419 & 20.7 & 3 & 10 & 47 & 95 & 1.14 \\
\hline 420 & 20.7 & 3 & 10 & 47 & 105 & 1.13 \\
\hline
\end{tabular}




\section{Appendix C. (Continued) Experimental Data (Prediction Set)}

\begin{tabular}{|r|r|r|r|r|r|r|}
\hline \hline $\mathrm{NO}$ & $\mathrm{P}$ & $\mathrm{IS}$ & $\mathrm{pH}$ & $\mathrm{PS}$ & $\mathrm{t}$ & $\mathrm{J}$ \\
\hline & $\mathrm{kPs}$ & $\mathrm{M}$ & & $\mathrm{nm}$ & $\mathrm{min}$ & $\mathrm{m} / \mathrm{s}$ \\
\hline 421 & 62.1 & 2 & 10 & 47 & 2 & 3.75 \\
\hline 422 & 62.1 & 2 & 10 & 47 & 7 & 2.9 \\
\hline 423 & 62.1 & 2 & 10 & 47 & 12 & 2.5 \\
\hline 424 & 62.1 & 2 & 10 & 47 & 17 & 2.2 \\
\hline 425 & 62.1 & 2 & 10 & 47 & 27 & 1.8 \\
\hline 426 & 62.1 & 2 & 10 & 47 & 32 & 1.68 \\
\hline 427 & 62.1 & 2 & 10 & 47 & 37 & 1.58 \\
\hline 428 & 62.1 & 2 & 10 & 47 & 47 & 1.43 \\
\hline 429 & 62.1 & 2 & 10 & 47 & 52 & 1.38 \\
\hline 430 & 62.1 & 2 & 10 & 47 & 57 & 1.3 \\
\hline 431 & 62.1 & 2 & 10 & 47 & 67 & 1.22 \\
\hline 432 & 62.1 & 2 & 10 & 47 & 72 & 1.2 \\
\hline 433 & 62.1 & 2 & 10 & 47 & 77 & 1.16 \\
\hline 434 & 62.1 & 2 & 10 & 47 & 95 & 1.08 \\
\hline 435 & 62.1 & 2 & 10 & 47 & 105 & 1.06 \\
\hline 436 & 41.4 & 2 & 10 & 47 & 0 & 2.8 \\
\hline 437 & 41.4 & 2 & 10 & 47 & 2 & 2.5 \\
\hline 438 & 41.4 & 2 & 10 & 47 & 7 & 2.2 \\
\hline 439 & 41.4 & 2 & 10 & 47 & 12 & 2 \\
\hline 440 & 41.4 & 2 & 10 & 47 & 17 & 1.8 \\
\hline 441 & 41.4 & 2 & 10 & 47 & 22 & 1.7 \\
\hline 442 & 41.4 & 2 & 10 & 47 & 27 & 1.58 \\
\hline 443 & 41.4 & 2 & 10 & 47 & 32 & 1.48 \\
\hline 444 & 41.4 & 2 & 10 & 47 & 37 & 1.38 \\
\hline 445 & 41.4 & 2 & 10 & 47 & 42 & 1.3 \\
\hline 446 & 41.4 & 2 & 10 & 47 & 47 & 1.28 \\
\hline 447 & 41.4 & 2 & 10 & 47 & 52 & 1.24 \\
\hline 448 & 41.4 & 2 & 10 & 47 & 57 & 1.23 \\
\hline 449 & 41.4 & 2 & 10 & 47 & 62 & 1.2 \\
\hline 450 & 41.4 & 2 & 10 & 47 & 67 & 1.16 \\
\hline 451 & 41.4 & 2 & 10 & 47 & 72 & 1.13 \\
\hline 452 & 41.4 & 2 & 10 & 47 & 77 & 1.1 \\
\hline 453 & 41.4 & 2 & 10 & 47 & 85 & 1.08 \\
\hline 454 & 41.4 & 2 & 10 & 47 & 95 & 1 \\
\hline 455 & 41.4 & 2 & 10 & 47 & 105 & 0.98 \\
\hline
\end{tabular}

\begin{tabular}{|r|r|r|r|r|r|r|}
\hline NO & $\mathrm{P}$ & $\mathrm{IS}$ & $\mathrm{pH}$ & $\mathrm{PS}$ & $\mathrm{t}$ & $\mathrm{J}$ \\
\hline & $\mathrm{kPs}$ & $\mathrm{M}$ & & $\mathrm{nm}$ & $\mathrm{min}$ & $\mathrm{m} / \mathrm{s}$ \\
\hline 456 & 41.4 & 2 & 10 & 47 & 115 & 0.95 \\
\hline 457 & 20.7 & 2 & 10 & 47 & 2 & 1.38 \\
\hline 458 & 20.7 & 2 & 10 & 47 & 7 & 1.25 \\
\hline 459 & 20.7 & 2 & 10 & 47 & 12 & 1.18 \\
\hline 460 & 20.7 & 2 & 10 & 47 & 17 & 1.14 \\
\hline 461 & 20.7 & 2 & 10 & 47 & 27 & 1.05 \\
\hline 462 & 20.7 & 2 & 10 & 47 & 32 & 1.01 \\
\hline 463 & 20.7 & 2 & 10 & 47 & 37 & 1 \\
\hline 464 & 20.7 & 2 & 10 & 47 & 47 & 0.95 \\
\hline 465 & 20.7 & 2 & 10 & 47 & 52 & 0.93 \\
\hline 466 & 20.7 & 2 & 10 & 47 & 57 & 0.92 \\
\hline 467 & 20.7 & 2 & 10 & 47 & 67 & 0.9 \\
\hline 468 & 20.7 & 2 & 10 & 47 & 72 & 0.89 \\
\hline 469 & 20.7 & 2 & 10 & 47 & 77 & 0.88 \\
\hline 470 & 20.7 & 2 & 10 & 47 & 95 & 0.85 \\
\hline 471 & 20.7 & 2 & 10 & 47 & 105 & 0.83 \\
\hline
\end{tabular}




\section{APPENDIX D. MATLAB CODE (RBF.M)}

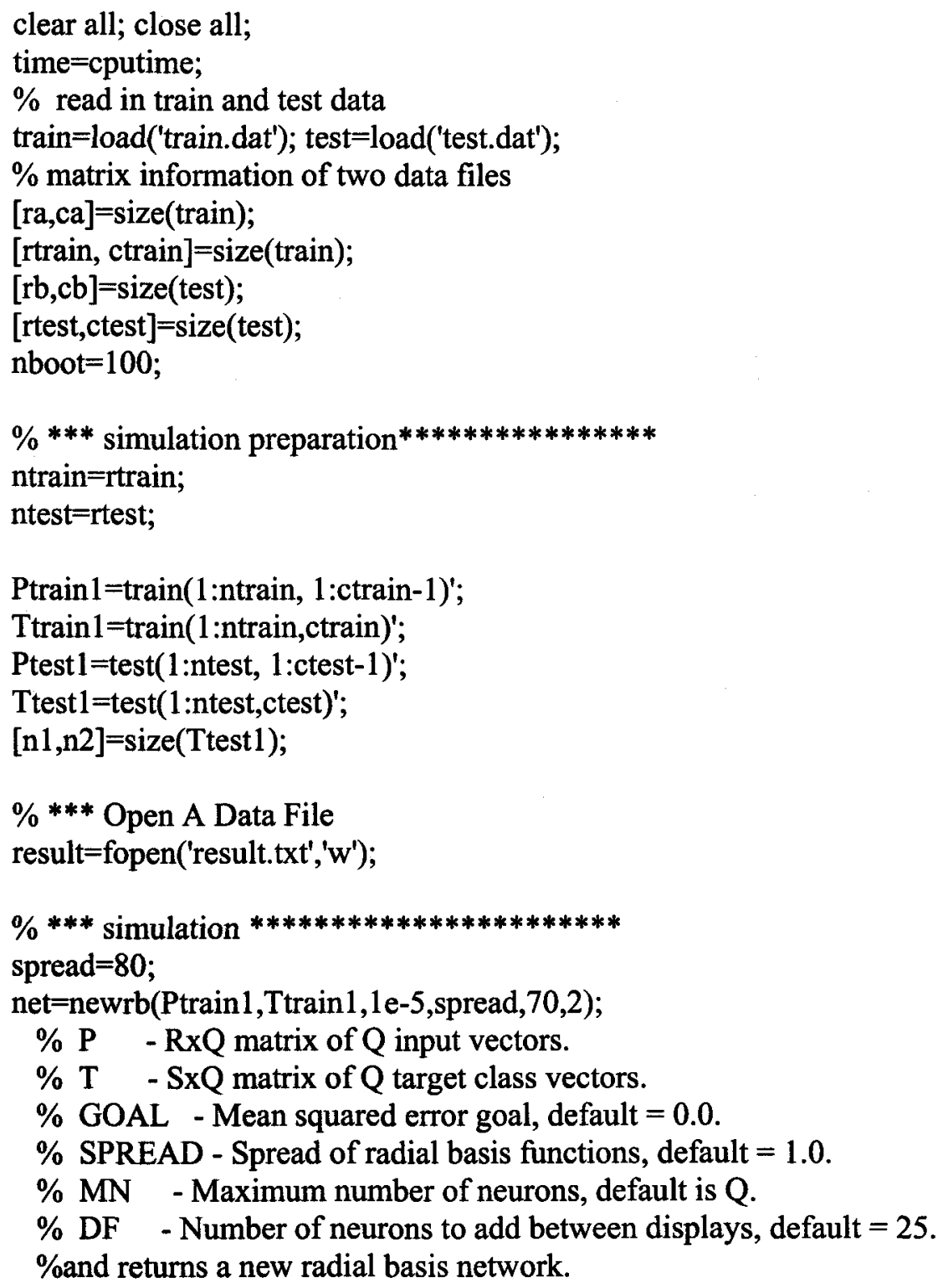




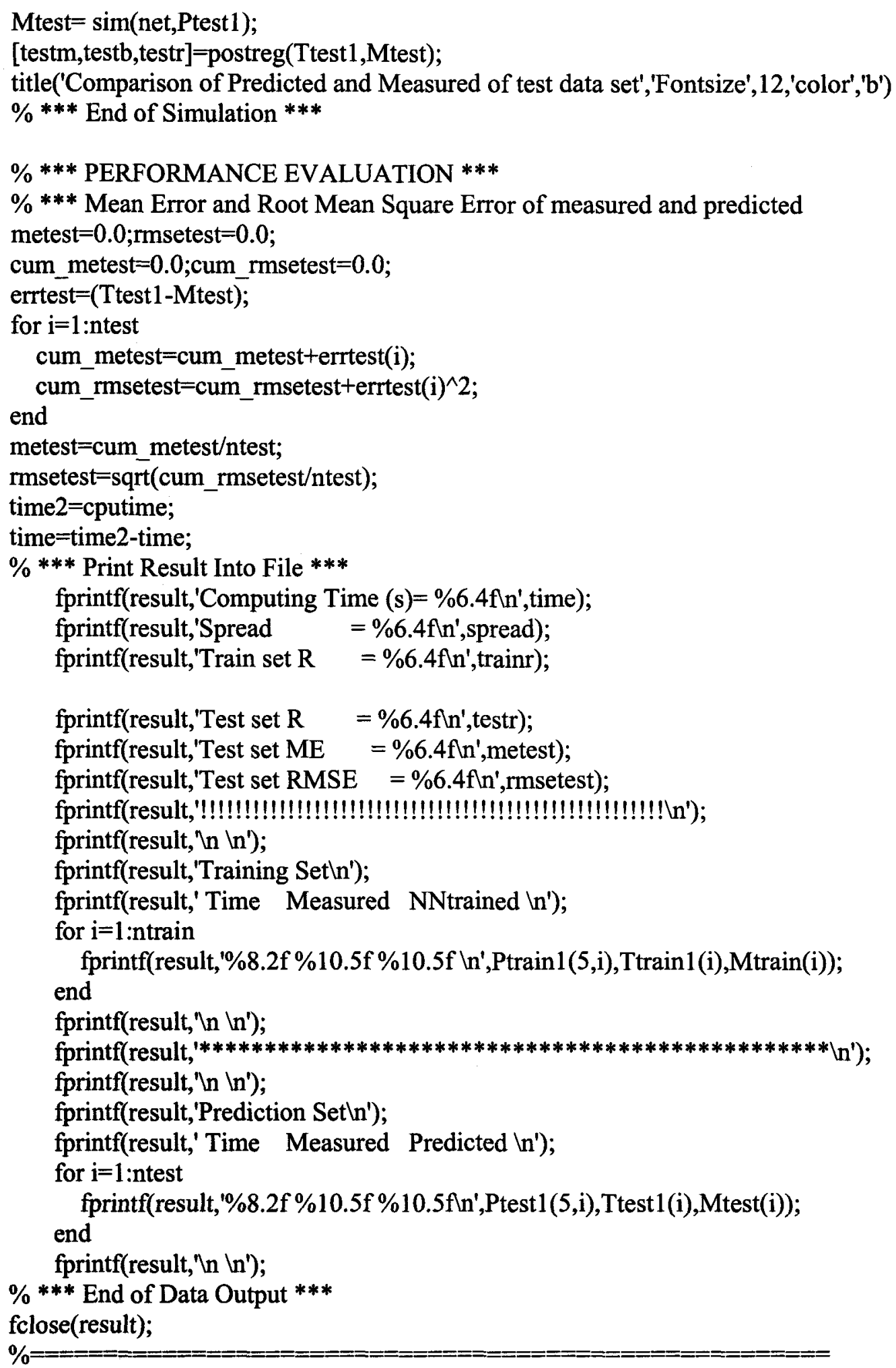




\section{APPENDIX E. MATLAB CODE (BPNN.M)}

clear all;

time=cputime;

$\%$ read in train and test data

train=load('train.dat');

test=load('test.dat');

$\%$ matrix information of two data files

[rtrain, ctrain] $=$ size(train);

[rtest,ctest] $=$ size(test);

$\%$ parameters

$\mathrm{m} 2=$ ctrain $-1 ; \mathrm{m} 1=$ ctrain;

$\mathrm{m} 4=400$;

$\mathrm{m} 5=25$;

$\mathrm{m} 6=1 \mathrm{e}-10$

nboot $=16$;

ntrain=rtrain;

ntest=rtest;

$\% * * *$ simulation $* *$

Ptrain=train(1:ntrain, 1:ctrain-1)';

Ttrain=train(1:ntrain,ctrain)';

Ptest=test(1:ntest, 1:ctest-1)';

Ttest=test(1:ntest,ctest)';

$[\mathrm{n} 1, \mathrm{n} 2]=$ size(Ttest);

$\mathrm{T} 1=\operatorname{zeros}(\mathrm{n} 2,2)$;

T1(:,1)=Ttest';

$\%$ open a file for result

result=fopen('result.txt','w');

$\%$ it makes sense to make a loop here to do more than one nn run

prev_testr $=0.0$;

prev_tme $=1.0$

testr $=0.0$

tme $=1.0$

for $n b=1:$ nboot

if testr>prev_testr

prev_testr=testr; 


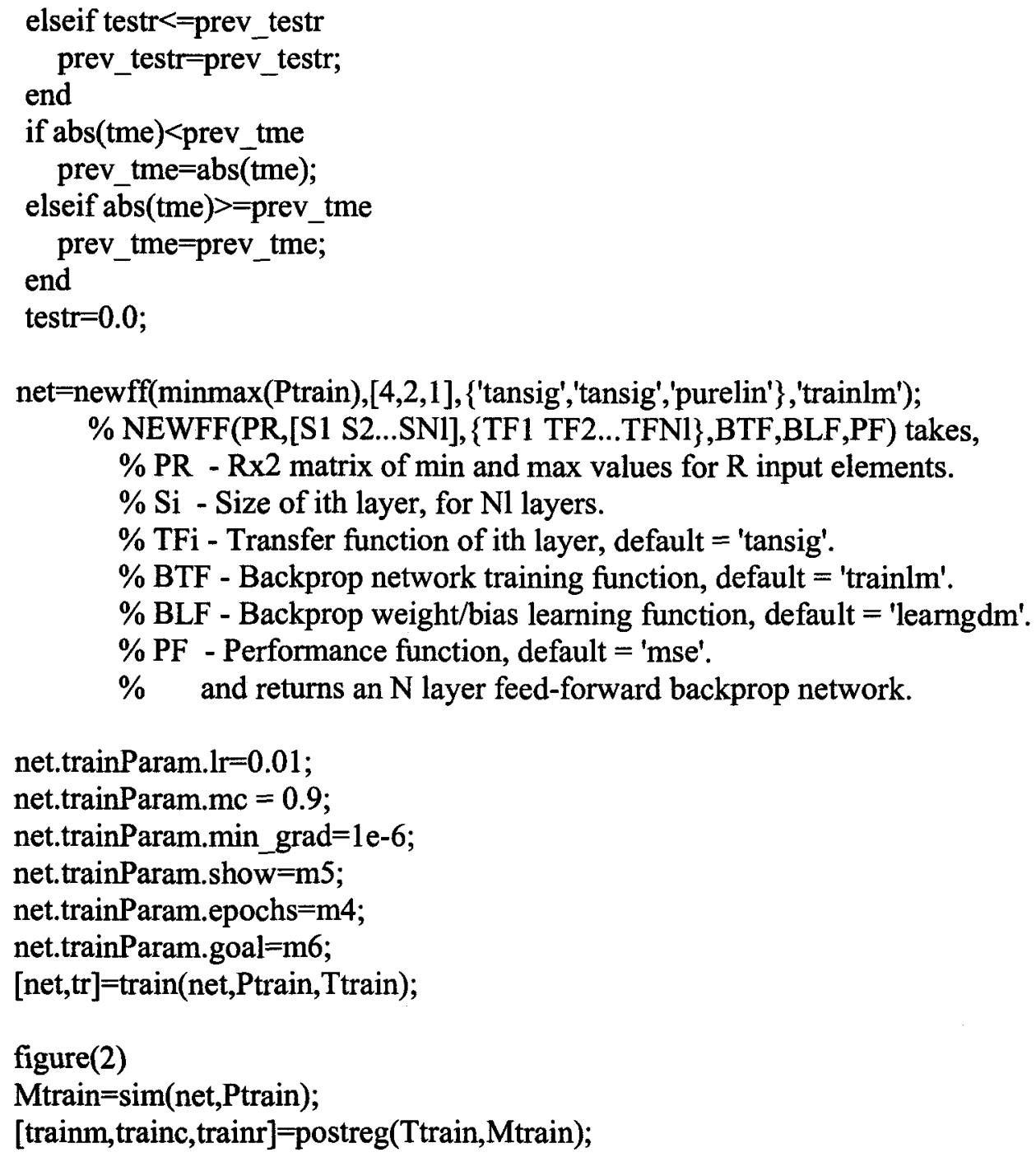




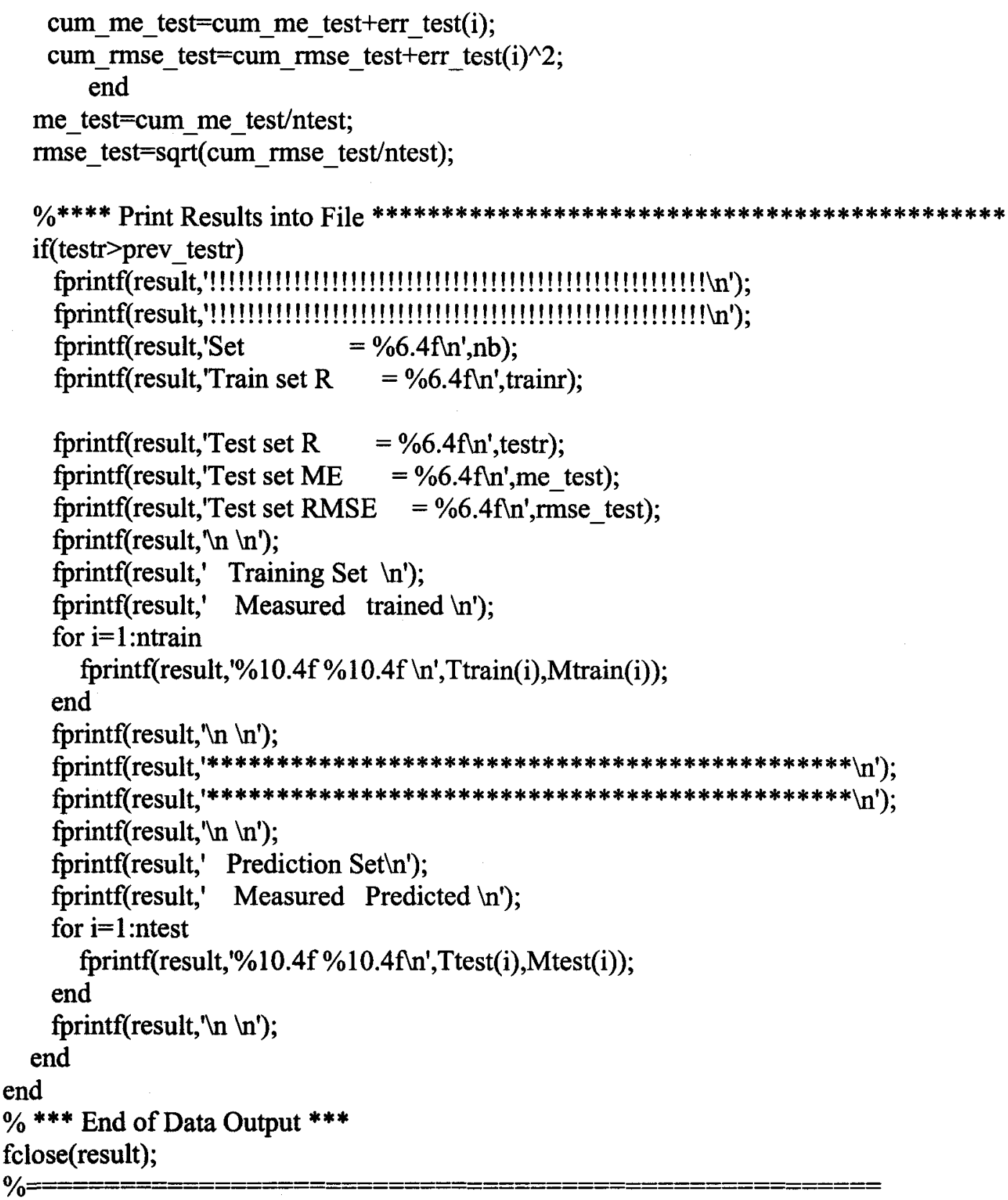




\section{APPENDIX F. MATLAB CODE (MULREG.M)}

clear all; close all;

time=cputime;

$\%$ read in train and test data

train=load('train.txt');

test=load('test.txt');

[rtrain, ctrain] =size(train);

[rtest,ctest] $=$ size(test);

$\% * * *$ simulation $* * * * * * * * * * * * * * * *$

ntrain=rtrain;

ntest=rtest;

nsamp $=$ ntrain + ntest;

[nsamp ntest ntrain]

Ptrain 1=train(1:ntrain, 1:ctrain-1);

$\mathrm{X}=$ [ones(ntrain,1) Ptrain1];

Ttrain $1=$ train(1:ntrain,ctrain);

Ptest $1=$ test( 1 :ntest, 1 :ctest-1);

$\mathrm{X} 1=$ [ones(ntest,1) Ptest1];

Ttest $1=$ test $(1$ :ntest,ctest);

$\%$ result data file

result=fopen('result.txt','w');

$\% * * *$ multiple regression $* * *$

a1=X $\backslash$ Ttrain 1

$\mathrm{Y}=\mathrm{X}^{*} \mathrm{a} 1$

$\mathrm{Y} 1=\mathrm{X} 1 * \mathrm{a} 1$

$\% * * *$ End of multiple regression $* * * *$

$\% * * * * * * * * * * *$ PERFORMANCE EVALUATION

MaxErrl $=\max ($ abs $(Y-T \operatorname{train} 1))$

MaxErr2=max (abs(Y1-Ttest1))

$\mathrm{rl}=\operatorname{corrcoef}(\mathrm{Y}, \mathrm{Ttrain} 1)$;

$\mathrm{r} 2=\operatorname{corrcoef}(\mathrm{Y} 1$, Ttest 1$)$;

$\%$ mse and rmse for training set 


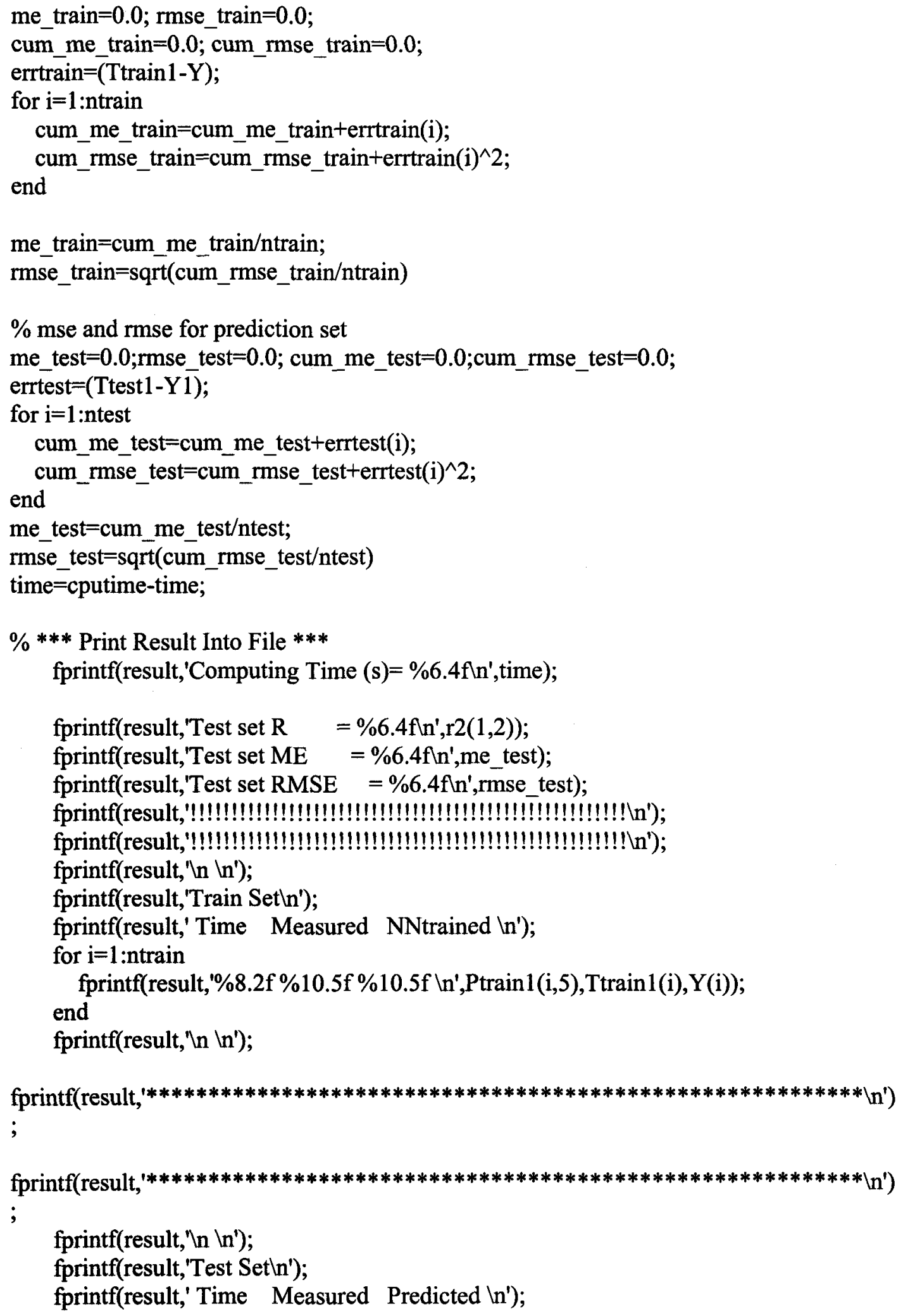




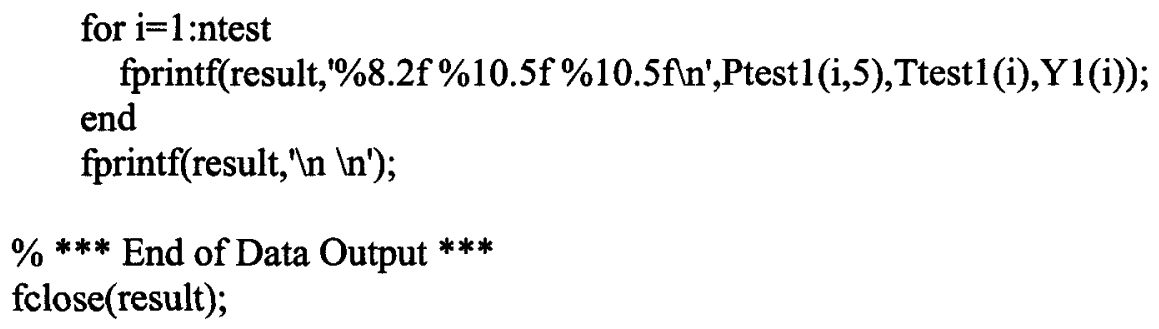

$\%==$ 


\section{References}

Alldrin, Neil, Andrew Smith and Doug Turnbull (2004) Classifying facial expression with radial basis function networks, using gradient descent and $k$-mean. On line: http://www.cs.ucsd.edu/ dturnbul/Papers/RBFproject.pdf

ASCE Task Committee on Application of Neural Networks in Hydrology (2000). Artificial neural networks in hydrology. I: Preliminary concepts. Journal of Hydrology 5(2): 115-123.

Bacchin, P., P. Aimar and V. Sanchez (1996). Influence of surface interaction on transfer during colloid ultrafiltration. Journal of Membrane Science 115(1): 49-63.

Bacchin, P., D. Si-Hassen, V. Starov, M.J. Clifton and P Aimar (2002). A unifying model for concentration polarization, gel-layer formation and particle deposition in cross-flow membrane filtration of colloidal suspensions. Chemical Engineering Science 57(1): 77-91.

Bowden, Gavin J., Graeme C. Dandy and Holger R. Maier (2005). Input determination for neural network models in water resources applications. Part 1--background and methodology. Journal of Hydrology 301(1-4): 75-92. 
Bowden, Gavin J., Holger R. Maier and Graeme C. Dandy (2005). Input determination for neural network models in water resources applications. Part 2. Case study: Forecasting salinity in a river. Journal of Hydrology 301(1-4): 93-107.

Bowen, W. Richard and Frank Jenner (1995). Theoretical descriptions of membrane filtration of colloids and fine particles: An assessment and review. Advances in Colloid and Interface Science 56: 141-200.

Bowen, W. Richard, Meirion G. Jones and Haitham N. S. Yousef (1998). Dynamic ultrafiltration of proteins - a neural network approach. Journal of Membrane Science 146(2): $225-235$.

Chakraborty, Mousumi, Chiranjib Bhattacharya and Siddhartha Dutta (2003). Studies on the applicability of artificial neural network (ANN) in emulsion liquid membranes. Journal of Membrane Science 220(1-2): 155-164.

Demuth, Howard and Mark Beale (2003). Neural network toolbox: For use with $M A T L A B$. User guide of The MathWorks.

Dornier, M., M. Decloux, G. Trystram and A. Lebert (1995). Dynamic modeling of crossflow microfiltration using neural networks. Journal of Membrane Science 98(3): 263-273. 
Faibish, Ron S., Menachem Elimelech and Yoram Cohen (1998). Effect of interparticle electrostatic double layer interactions on permeate flux decline in crossflow membrane filtration of colloidal suspensions: An experimental investigation. Journal of Colloid and Interface Science 204(1): 77-86.

Fausett, Laurene (1994). Fundamentals of neural networks. Englewood Cliffs, Prentice Hall.

Kim, Albert S. (2004). Classnote on membrane separation. Department of Civil and Environmental Engineering, University of Hawai'i at Manoa.

Maas, Olivier, Jean-Philippe Boulanger and Sylvie Thiria (2000). Use of neural networks for predictions using time series: Illustration with the El Nino Southern Oscillation phenomenon. Neurocomputing 30(1-4): 53-58.

Mallevialle, Joel , Peter E. Odendaal and Mark R. Wiesner (1996). Water treatment membrane processes, McGraw Hill.

Mcdonogh, Richard M., Anthony G. Fane and Christopher J. D. Fell (1989). Charge effects in the cross-flow filtration of colloids and particulates. Journal of Membrane Science 43(1): 69-85. 
Mulder, Marcel (1997). Basic principles of membrane technology, Kluwer Academic Publishers.

Niemi, Harri, Abhay Bulsari and Seppo Palosaari (1995). Simulation of membrane separation by neural networks. Journal of Membrane Science 102: 185-191.

Ramaswamy, Senthilkumar, Alan R. Greenberg and Michael L. Peterson (2004). Noninvasive measurement of membrane morphology via UFDR: Pore-size characterization. Journal of Membrane Science 239(1): 143-154.

Razavi, Mohammad A., Ali Mortazavi and Mahmoud Mousavi (2003). Dynamic modelling of milk ultrafiltration by artificial neural network. Journal of Membrane Science 220(1-2): 47-58.

Romero, Cecily A. and Robert H. Davis (1988). Global model of crossflow microfiltration based on hydrodynamic particle diffusion. Journal of Membrane Science 39(2): 157-185.

Romero, Cecily A. and Robert H. Davis (1990). Transient model of crossflow microfiltration. Chemical Engineering Science 45(1): 13-25. 
Sahoo, Goloka B., Chittaranjan Ray and H. F. Wade (2005). Pesticide prediction in ground water in North Carolina domestic wells using artificial neural networks. Journal Ecological Modelling 183: 29-46.

Schilling, Robert J., James J. Carroll and Ahmad F. Al-Ajlouni (2001). Approximation of nonlinear systems with radial basis function neural networks. Transactions on neural networks 12(1): 1-15.

Shetty, Grishma R., Heidar Malki and Shankararaman Chellam (2003). Predicting contaminant removal during municipal drinking water nanofiltration using artificial neural networks. Journal of Membrane Science 212(1-2): 99-112.

Song, L. and Elimelech M (1995). Theory of concentration polarization in crossflow filtration. Journal of the Chemical Society, Faraday Transactions 91: 3389-3398.

Valverde Ramirez, Maria Cleofe, Haroldo Fraga de Campos Velho and Nelson Jesus Ferreira (2005). Artificial neural network technique for rainfall forecasting applied to the Sao Paulo region. Journal of Hydrology 301(1-4): 146-162.

Wilks, Daniel S. (1995). Statistical methods in the atmospheric sciences, Academic Press. 